University of Nebraska - Lincoln

DigitalCommons@University of Nebraska - Lincoln

Anthony F. Starace Publications

Research Papers in Physics and Astronomy

$10-30-2017$

\title{
Doubly-excited state effects on two-photon double ionization of helium by time-delayed, oppositely circularly-polarized attosecond pulses
}

Jean Marcel Ngoko Djiokap

University of Nebraska-Lincoln, marcelngoko@unl.edu

Anthony F. Starace

University of Nebraska-Lincoln, astarace1@unl.edu

Follow this and additional works at: http:// digitalcommons.unl.edu/physicsstarace

Part of the Atomic, Molecular and Optical Physics Commons, Elementary Particles and Fields and String Theory Commons, and the Plasma and Beam Physics Commons

Ngoko Djiokap, Jean Marcel and Starace, Anthony F., "Doubly-excited state effects on two-photon double ionization of helium by time-delayed, oppositely circularly-polarized attosecond pulses" (2017). Anthony F. Starace Publications. 227.

http://digitalcommons.unl.edu/physicsstarace/227

This Article is brought to you for free and open access by the Research Papers in Physics and Astronomy at DigitalCommons@University of Nebraska Lincoln. It has been accepted for inclusion in Anthony F. Starace Publications by an authorized administrator of DigitalCommons@University of Nebraska - Lincoln. 


\section{PAPER}

Doubly-excited state effects on two-photon double ionization of helium by time-delayed, oppositely circularly-polarized attosecond pulses

To cite this article: J M Ngoko Djiokap and Anthony F Starace 2017 J. Opt. 19124003

View the article online for updates and enhancements. 


\title{
Doubly-excited state effects on two-photon double ionization of helium by time-delayed, oppositely circularly-polarized attosecond pulses
}

\author{
J M Ngoko Djiokap (i) and Anthony F Starace \\ Department of Physics and Astronomy, The University of Nebraska, Lincoln, NE 68588-0299, United \\ States of America \\ E-mail: marcelngoko@unl.edu and astarace1@unl.edu
}

Received 5 July 2017, revised 11 September 2017

Accepted for publication 28 September 2017

Published 30 October 2017

\begin{abstract}
We study two-photon double ionization (TPDI) of helium by a pair of time-delayed (nonoverlapping), oppositely circularly-polarized attosecond pulses whose carrier frequencies are resonant with ${ }^{1} \mathrm{P}^{o}$ doubly-excited states. All of our TPDI results are obtained by numerical solution of the two-electron time-dependent Schrödinger equation for the six-dimensional case of circularly-polarized attosecond pulses, and they are analyzed using perturbation theory (PT). As compared with the corresponding nonresonant TPDI process, we find that the doubly-excited states change the character of vortex patterns in the two-electron momentum distributions for the case of back-to-back detection of the two ionized electrons in the polarization plane. The doublyexcited states also completely change the structure of fixed-energy, two-electron angular distributions. Moreover, both the fixed-energy and energy-integrated angular distributions, as well as the two-electron energy distributions, exhibit a periodicity with time delay $\tau$ between the two attosecond pulses of about 69 as, i.e. the beat period between the $(2 s 2 p){ }^{1} \mathrm{P}^{o}$ doubly-excited state and the He ground state. Using PT we derive an expression for an angle-integrated energy distribution that is sensitive to the slower beat period $\sim 1.2 \mathrm{fs}$ between different doubly-excited states as well as to the long timescale $\sim 17$ fs of autoionization lifetimes. However, with our current computer codes we are only able to study numerically the time-dependent phenomena occurring on an attosecond time scale.
\end{abstract}

Keywords: two-photon double ionization, circularly-polarized attosecond pulses, electron matter-wave vortices, doubly-excited state dynamics, He atom, quantum beats, multipath ionization interferometry

(Some figures may appear in colour only in the online journal)

\section{Introduction}

Since 2001 the experimental realizations of attosecond pulse trains [1] and of isolated attosecond pulses of increasingly shorter pulse durations [2-5] have stimulated the growth of attosecond physics [6]. A main goal of this emerging new field is to image and control electronic motions inside atoms and molecules on their characteristic attosecond time scales (where 1 attosecond $=10^{-18} \mathrm{~s}$ ). Pump-probe processes with isolated attosecond pulses (which have been dubbed the "holy grail' of attosecond science [7]) would permit very precise timing of electronic processes in matter. The potential of such pump-probe processes has been demonstrated experimentally on a time scale of $\approx 1$ fs for the case of two-photon double ionization (TPDI) of the xenon atom by a pair of timedelayed, broadband extreme ultraviolet (XUV) pulses 

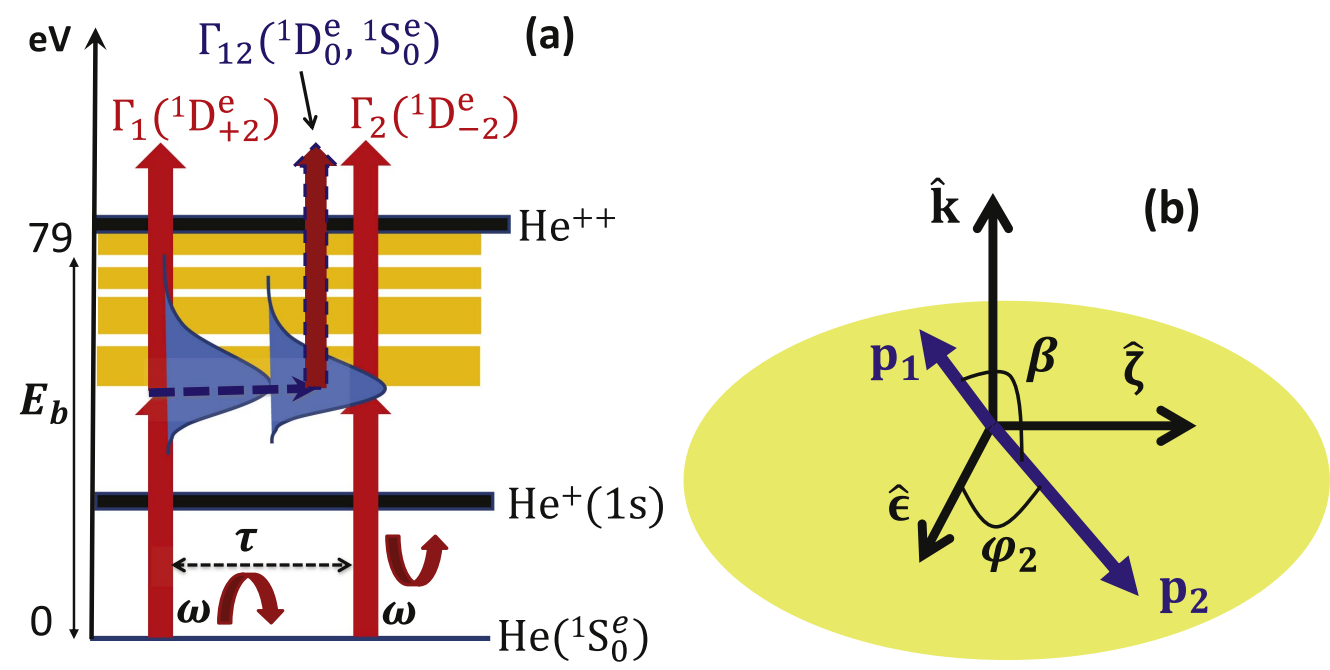

Figure 1. (a) Schematic energy-level diagram for direct TPDI of He (with binding energy $E_{b}=79 \mathrm{eV}$ ) by two non-overlapping counterrotating circularly-polarized pulses delayed in time by $\tau$. Each pulse has a carrier frequency $\omega=60 \mathrm{eV}$. The two direct TPDI pathways, $\Gamma_{1}\left({ }^{1} S_{0}^{e} \rightarrow{ }^{1} \mathrm{D}_{+2}^{e}\right)$ and $\Gamma_{2}\left({ }^{1} \mathrm{~S}_{0}^{e} \rightarrow{ }^{1} \mathrm{D}_{-2}^{e}\right)$, correspond to two-photon absorption from each pulse (where the subscripts on the term levels indicate the $M_{L}$ values). The TPDI pathway $\Gamma_{12}\left({ }^{1} \mathrm{~S}_{0}^{e} \rightarrow{ }^{1} \mathrm{D}_{0}^{e},{ }^{1} \mathrm{~S}_{0}^{e}\right)$ corresponds to one-photon absorption from each pulse, where the first pulse coherently excites a manifold of doubly-excited states (located in the shaded (orange) region), followed by a second pulse that probes the ensuing electronic motion. Note that sequential TPDI channels (not shown for simplicity) are also present, but they cannot be distinguished from the direct TPDI channels by a single pulse. (b) Detection geometry for back-to-back emission of electron momenta $\mathbf{p}_{1,2}$ in the polarization plane $(\hat{\epsilon}, \hat{\zeta})$, where $\hat{\epsilon}$ and $\hat{\zeta}=\hat{\mathbf{k}} \times \hat{\epsilon}$ are respectively the major and minor axes of the polarization ellipse, and $\hat{\mathbf{k}} \| \hat{\mathbf{z}}$ is the propagation direction of each pulse.

resonant with Xe doubly-excited states $[8]^{1}$. However, such measurements are not common owing to the relatively weak intensity of existing isolated attosecond pulses [9]. Nevertheless, numerous theoretical studies of time-dependent electron motion in the helium atom have been carried out in which an attosecond pump pulse produces a coherent superposition of excited states followed, after a time delay, by an attosecond probe pulse that doubly-ionizes the excited $\mathrm{He}$ atom (see e.g. $[8]^{1}$ and $[10-13]$ ). Nearly all of these theoretical investigations show how the beat periods of the coherent superposition of intermediate states, both among themselves and with the ground state, affects the $\mathrm{He}^{2+}$ yield. Note that all of the above-cited experimental and theoretical works have involved linearly-polarized attosecond pulses. However, recently methods have been proposed for producing short, coherent XUV pulses with tunable polarization (see e.g. $[14,15]$ and references therein).

Recent theoretical studies have shown that ellipticallypolarized attosecond pulses can produce novel phenomena [16-21] that do not occur for linearly-polarized pulses. One such phenomenon is a new nonlinear dichroic effect in double ionization of He by elliptically-polarized attosecond pulses [16]. Another is the prediction of electron matter-wave vortex patterns in the ionized electron momentum distribution following single ionization of He by counter-rotating circularly-polarized attosecond pulses [17, 19]. In particular, it was predicted in [19] that the number of spiral arms in the vortex pattern depends on the number of photons required for single electron ionization.

\footnotetext{
1 See the supplementary information of [8] for a theoretical study of twophoton double ionization of the He atom by a pair of time-delayed attosecond pulses resonant with the region of doubly-excited states.
}

These predictions have recently been confirmed experimentally for single ionization of potassium atoms using circularlypolarized femtosecond pulses [22]. Specifically, in [22] it was shown that in three-photon ionization of the $\mathrm{K}$ atom ground state, the vortex pattern has six spiral arms, whereas in the case that population is transferred by an intense $\pi$-pulse from the ground state to a resonant excited state requiring only two photons for ionization, then the vortex pattern has only four spiral arms. Such matter-wave vortices have also been predicted in single ionization of the $\mathrm{H}_{2}^{+}$and $\mathrm{H}_{3}^{2+}$ molecules $[18,20]$. Recently, vortex patterns have been predicted in the two-electron momentum distributions produced by single-photon double ionization of $\mathrm{He}$ [21].

In this paper, we study the correlated process of singlecolor TPDI of He via doubly-excited states by a pair of timedelayed (non-overlapping), counter-rotating circularly-polarized attosecond XUV pulses (see figure 1(a)). Our numerical results are obtained by solving the two-electron time-dependent Schrödinger equation (TDSE) in six spatial dimensions, and they are analyzed using perturbation theory (PT). Our study is similar to those in $[8]^{1}$ and [12] except that we employ circularly-polarized pulses rather than linearly-polarized pulses. Moreover, rather than investigating the TPDI yield, we study different observables, e.g. the two-electron momentum, angular, and energy distributions, each of which is shown to be strongly affected by the resonant doubly-excited states. Our numerical results exhibit quantum beats between the ground state and doubly-excited states (QB-I) with a period of $\sim 69$ as. In addition, our PT analysis also predicts quantum beats between autoionizing doubly-excited states (QB-II), with a period of $\sim 1.2 \mathrm{fs}$. 
This paper is organized as follows. In the second section, we briefly present the computational method. In the third section, we give a short account of our PT formulation. In the fourth section, our TDSE results for the momentum, angular and energy distributions are analyzed using PT. In the last section, we summarize our results and conclusions. We employ atomic units $\left(e=m_{e}=\hbar=1\right)$ unless specified otherwise.

\section{Computational method}

To explore doubly-excited state dynamics in TPDI of $\mathrm{He}$ in the momentum, angular, and energy distributions, we solve the two-electron TDSE in six spatial dimensions within the length gauge and the dipole approximation for He interacting with a pair of time-delayed, oppositely circularly-polarized attosecond pulses. The two pulses have the same carrier frequency $\omega$ and intensity $I=F_{0}^{2}$, where $F_{0}$ is the electric field strength. The electric field $\mathbf{F}(t)$ of such a pair of pulses is,

$$
\begin{aligned}
\boldsymbol{F}(t)= & \boldsymbol{F}_{1}(t)+\boldsymbol{F}_{2}(t-\tau) \equiv F_{0}^{(1)}(t) \operatorname{Re}\left[\mathbf{e}_{1} \mathrm{e}^{-\mathrm{i}\left(\omega t+\phi_{1}\right)}\right] \\
& +F_{0}^{(2)}(t-\tau) \operatorname{Re}\left[\mathbf{e}_{2} \mathrm{e}^{-\mathrm{i}\left(\omega(t-\tau)+\phi_{2}\right)}\right],
\end{aligned}
$$

where for the $j$ th pulse $(j=1,2) \mathbf{e}_{j}$ is its polarization, $\phi_{j}$ is its carrier-envelope-phase (CEP), $F_{0}^{(j)}(t) / F_{0}=\cos ^{2}\left(\pi t / T_{j}\right)$ is its temporal envelope, and $T_{j}=n_{j}(2 \pi / \omega)$ gives its pulse duration, where $n_{j}$ is the number of cycles. We define the polarization vector of the $j$ th pulse by:

$$
\mathbf{e}_{j} \equiv\left(\hat{\boldsymbol{\epsilon}}+\mathrm{i} \eta_{j} \hat{\zeta}\right) / \sqrt{1+\eta_{j}^{2}},
$$

where $\hat{\boldsymbol{\epsilon}}$ and $\hat{\boldsymbol{\zeta}} \equiv \hat{\mathbf{k}} \times \hat{\boldsymbol{\epsilon}}$ are respectively the major and minor axes of the polarization ellipse, $\hat{\boldsymbol{k}} \| \hat{\mathbf{z}}$ is the pulse propagation direction, and $\eta_{j}$ is the ellipticity $\left(-1 \leqslant \eta_{j} \leqslant+1\right)$. The degrees of linear and circular polarization of the $j$ th pulse are defined respectively by $\ell_{j} \equiv\left(\mathbf{e}_{j} \cdot \mathbf{e}_{j}\right)=\left(1-\eta_{j}^{2}\right) /\left(1+\eta_{j}^{2}\right)$ and $\xi_{j} \equiv \operatorname{Im}\left[\mathbf{e}_{j}^{*} \times \mathbf{e}_{j}\right]_{z}=2 \eta_{j} /\left(1+\eta_{j}^{2}\right)$.

The numerical methods used to solve the TDSE for the electric field (1) are described elsewhere [17, 19, 21]. In brief, we employ a time-dependent close-coupling expansion $[23,24]$ of the wave packet $\Psi\left(\mathbf{r}_{1}, \mathbf{r}_{2} ; t\right)$ onto the orthonormal basis functions of bipolar-spherical harmonics $\Lambda_{l_{1}, l_{2}}^{L M}\left(\hat{\mathbf{r}}_{1}, \hat{\mathbf{r}}_{2}\right)$, where $L$ is the total angular momentum of the two-electron system, $M$ is its azimuthal quantum number, and $l_{1}, l_{2}$ are the individual electron orbital angular momenta. The twoelectron TDSE in six spatial dimensions is solved using a finite-element discrete-variable representation [25] combined with the real-space-product algorithm (a split-operator method) [26-29] together with Wigner rotation transformations at each time step from the laboratory frame to the frame of the instantaneous electric field [30, 31]. At the end of the two pulses, i.e. at $t=T_{f}=T+\tau$, we freely propagate the two-electron wave packet $\Psi\left(\mathbf{r}_{1}, \mathbf{r}_{2} ; t\right)$ for a time $T_{p}$ before extracting the six-fold differential probability (SDP) [32] for TPDI of He by projecting the two-electron continuum part, $\Psi^{\prime}\left(\mathbf{r}_{1}, \mathbf{r}_{2} ; T_{f}+T_{p}\right)$, of the wave packet $\Psi\left(\mathbf{r}_{1}, \mathbf{r}_{2} ; T_{f}+T_{p}\right)$ onto the double-continuum final state, which is approximated by a product of two Coulomb waves $\Phi_{\mathbf{p}_{1}, \mathbf{p}_{2}}^{(-)}\left(\mathbf{r}_{1}, \mathbf{r}_{2}\right)$ with charge $Z=2$. The SDP, $\mathcal{W}_{\xi_{2}}^{\xi_{1}}\left(\mathbf{p}_{1}, \mathbf{p}_{2} ; \tau\right)$, for producing two continuum electrons with momenta $\mathbf{p}_{1}$ and $\mathbf{p}_{2}$ is thus:

$$
\mathcal{W}_{\xi_{2}}^{\xi_{1}}\left(\mathbf{p}_{1}, \mathbf{p}_{2} ; \tau\right)=\left|\left\langle\Phi_{\mathbf{p}_{1}, \mathbf{p}_{2}}^{(-)}\left(\mathbf{r}_{1}, \mathbf{r}_{2}\right) \mid \Psi^{\prime}\left(\mathbf{r}_{1}, \mathbf{r}_{2}, T_{f}+T_{p}\right)\right\rangle\right|^{2} .
$$

Our calculations include four total angular momenta $(L=0-3)$, their azimuthal quantum numbers $|M| \leqslant L$, all combinations of individual electron orbital angular momenta $l_{1}, l_{2}=0-5$, and their azimuthal quantum numbers $\left|m_{1}\right| \leqslant l_{1}$ and $\left|m_{2}\right| \leqslant l_{2}$. We have checked that the $L=3$ angular momentum states play only a very minor role for the pulse parameters employed in our calculations. We note that the same number of angular momentum states have been used in TPDI of He with linearly polarized photons (with $\omega=60 \mathrm{eV}$ ) by Foumouo et al [33]. They also found that three photon processes play a negligible role for pulse intensities up to $10^{14} \mathrm{~W} \mathrm{~cm}^{-2}$.

\section{PT for TPDI of helium by non-overlapping pulses}

Achieving temporal coherent control of the process of TPDI of He via doubly-excited states using circularly-polarized pulses (see figure 1(a)) depends critically on the pulse parameters. First, the carrier frequency $\omega=60 \mathrm{eV}$ of the two pulses is chosen to be on resonance with the transition between the ground state and the $(2 s 2 p){ }^{1} \mathrm{P}^{o}$ doubly-excited state. Second, the bandwidth $\Delta \omega$ of the first pulse should be quite broad such that the created wavepacket encompasses several doubly-excited states. However, it should not be so broad as to allow double ionization of the helium atom (with a ground state binding energy of $E_{b}=79 \mathrm{eV}$ ) by a singlephoton transition. These two conditions are satisfied by choosing $n_{1}=6$ optical cycles for each pulse, which gives a bandwidth of $\Delta \omega \simeq 1.44 \omega / n_{1}=14.4 \mathrm{eV}$ for a cosinesquared envelope, and a pulse duration of $T_{r}=413.6$ as. Third, the intensity of the first pulse is $10^{14} \mathrm{~W} \mathrm{~cm}^{-2}$, which is intense enough to allow significant population to be transferred into doubly-excited states by a single-photon transition from the ground state, but is not so intense that nonlinear effects beyond the second-order occur. Finally, to explore doubly-excited state dynamics with different timescales (i.e. QB-I, QB-II, and autoionization) the time delay $\tau$ between the two pulses should be large enough that they do not overlap, i.e. $\tau \geqslant T_{r}$. For such pulse parameters, the ponderomotive energy is $U_{p}=3.98 \mathrm{meV}$, which is much smaller than $\omega$; thus PT is applicable. Moreover, for the intensity employed, the rotating wave approximation (RWA) is valid. Thus, it is legitimate to neglect photon emission processes in our PT analyses, which is equivalent to neglecting the complex conjugate terms in the electric field (1).

For oppositely circularly-polarized pulses, $\mathbf{e} \equiv \mathbf{e}_{1}=\mathbf{e}_{2}^{*}$, $\xi \equiv \xi_{1}=-\xi_{2}= \pm 1, \quad$ and $\quad \ell \equiv \ell_{1}=\ell_{2}=0$. The $\quad$ SDP $\mathcal{W}_{-\xi}^{\xi}\left(\mathbf{p}_{1}, \mathbf{p}_{2} ; \tau\right)$ for TPDI of He by a pair of oppositely 
circularly-polarized attosecond pulses delayed in time by $\tau$ is:

$$
\mathcal{W}_{-\xi}^{\xi}\left(\mathbf{p}_{1}, \mathbf{p}_{2} ; \tau\right)=\frac{\mathrm{d}^{6} W}{\mathrm{~d} \mathbf{p}_{1} \mathrm{~d} \mathbf{p}_{2}}=\left|A_{-\xi}^{\xi}\left(\mathbf{p}_{1}, \mathbf{p}_{2} ; \tau\right)\right|^{2},
$$

where $A_{-\xi}^{\xi}\left(\mathbf{p}_{1}, \mathbf{p}_{2} ; \tau\right)$ is the second-order PT transition amplitude. We neglect spin-orbit interactions; thus $A_{-\xi}^{\xi}\left(\mathbf{p}_{1}, \mathbf{p}_{2} ; \tau\right)$ is a scalar independent of the quantization axis. Therefore, $A_{-\xi}^{\xi}\left(\mathbf{p}_{1}, \mathbf{p}_{2} ; \tau\right)$ can be parameterized in terms of the vectors of the problem, namely, the polarization vectors $\mathbf{e}_{1}=\mathbf{e}$ and $\mathbf{e}_{2}=\mathbf{e}^{*}$, and the reduced two-electron momenta, $\mathbf{p}_{ \pm}=$ $\left(\hat{\mathbf{p}}_{1} \pm \hat{\mathbf{p}}_{2}\right) / 2$ [29], with coefficients that are scalar functions of $\rho \equiv\left(p_{1}, p_{2}, \beta\right)$, where $\cos \beta=\hat{\mathbf{p}}_{1} \cdot \hat{\mathbf{p}}_{2}$. We focus in this paper on the detection geometry for back-to-back (BTB) emission of electrons (see figure 1(b)), as it has been shown to be suitable for observing vortices in the SDP [21]. In the BTB detection geometry, one has $\mathbf{p}_{-}=\hat{\mathbf{p}}_{1}=-\hat{\mathbf{p}}_{2}$, so that $\mathbf{p}_{+}=0$. Using equations (6) and (7) in [29], the PT amplitude for the TPDI of $\mathrm{He}$ (under the RWA for our BTB detection geometry and for any energy sharing between the two electrons) takes the form:

$A_{-\xi}^{\xi}\left(\mathbf{p}_{-} ; \tau\right)=\mathrm{e}^{-2 \mathrm{i} \phi_{1}}\left[A_{\Gamma_{1}}\left(\mathbf{p}_{-}\right)+A_{\Gamma_{2}}\left(\mathbf{p}_{-} ; \tau\right)+A_{\Gamma_{12}}\left(\mathbf{p}_{-} ; \tau\right)\right]$,

where $\phi_{1}$ is the CEP of the first pulse, and

$$
\begin{gathered}
A_{\Gamma_{1}}\left(\mathbf{p}_{-}\right)=h_{-}(\rho)\left(\mathbf{p}_{-} \cdot \mathbf{e}\right)^{2}, \\
A_{\Gamma_{2}}\left(\mathbf{p}_{-} ; \tau\right) \simeq h_{-}(\rho)\left(\mathbf{p}_{-} \cdot \mathbf{e}^{*}\right)^{2} \mathrm{e}^{\mathrm{i} \Phi}, \\
A_{\Gamma_{12}}\left(\mathbf{p}_{-} ; \tau\right)=\left[h_{-}^{\prime}(\rho ; \tau) \mid \mathbf{p}_{-} \cdot \mathbf{e}^{2}+h^{\prime}(\rho ; \tau)\right] \mathrm{e}^{\mathrm{i} \psi} .
\end{gathered}
$$

In the PT amplitude (5), $A_{\Gamma_{1}}$ ( $\mathbf{p}_{-}$) defined by (6) corresponds to the path $\Gamma_{1}\left({ }^{1} \mathrm{~S}_{0}^{e} \rightarrow{ }^{1} \mathrm{D}_{+2}^{e}\right) ; A_{\Gamma_{2}}\left(\mathbf{p}_{-} ; \tau\right)$ defined by (7) corresponds to the path $\Gamma_{2}\left({ }^{1} \mathrm{~S}_{0}^{e} \rightarrow{ }^{1} \mathrm{D}_{-2}^{e}\right)$; and $A_{\Gamma_{12}}\left(\mathbf{p}_{-} ; \tau\right)$ defined by (8) corresponds to the path $\Gamma_{12}\left({ }^{1} S_{0}^{e} \rightarrow{ }^{1} D_{0}^{e},{ }^{1} S_{0}^{e}\right)$, where the three paths are shown in figure 1(a). In equations (6) and (7), the geometric factor $\left(\mathbf{p}_{-} \cdot \mathbf{e}\right)^{2}$ is

$$
\begin{aligned}
\left(\mathbf{p}_{-} \cdot \mathbf{e}\right)^{2} & =\frac{1}{2} \sin ^{2}(\beta / 2) \sin ^{2} \theta \mathrm{e}^{ \pm 2 i \varphi} \\
& \equiv \sqrt{8 \pi / 15} Y_{2, M}(\theta, \varphi) \sin ^{2}(\beta / 2),
\end{aligned}
$$

where $\theta$ and $\varphi$ are, respectively, the polar and azimuthal angles of the electron-pair momentum $\mathbf{p}_{-}$, and the azimuthal quantum number is $M=+2(-2)$ for right(left) circular polarization. In equation (8), the geometric factor $\left|\mathbf{p}_{-} \cdot \mathbf{e}\right|^{2}$ is given by

$$
\begin{aligned}
\left|\mathbf{p}_{-} \cdot \mathbf{e}\right|^{2} & =\frac{1}{2} \sin ^{2}(\beta / 2) \sin ^{2} \theta \\
& \equiv \frac{1}{6}\left[2-\sqrt{16 \pi / 5} Y_{2,0}(\theta, \varphi)\right] \sin ^{2}(\beta / 2) .
\end{aligned}
$$

In equation (7), it is assumed that the dynamical parameter for path $\Gamma_{2}$ is approximately the same as the one for path $\Gamma_{1}$, i.e. $h_{-}^{(2)}(\rho) \simeq h_{-}^{(1)}(\rho) \equiv h_{-}(\rho)$. From the geometric factor in equation (9), one sees that these channels lead to final states with $L=2, M^{\Gamma_{1}}=-M^{\Gamma_{2}}= \pm 2$, where $+(-)$ indicates right (left) circular polarization. From equation (8), one sees that the pump-probe channel $\Gamma_{12}$ has two terms. The first term, having the geometric factor $\left|\mathbf{p}_{-} \cdot \mathbf{e}\right|^{2}(10)$ and the dynamical parameter $h_{-}^{\prime}(\rho ; \tau)$, leads to final states with $L=0,2, M=0$. The scalar second term, having the dynamical parameter $h^{\prime}(\rho ; \tau)$, leads to final states with $L=0, M=0$. Parity conservation and particle exchange rules require that in equations (6)-(8) the ellipticity-independent parameters $h_{-}(\rho), h^{\prime}(\rho ; \tau)$, and $h^{\prime}{ }_{-}(\rho ; \tau)$ (where $\rho \equiv\left(p_{1}, p_{2}, \beta\right)$ ) are symmetric under the exchange of electron momenta $\mathbf{p}_{1} \leftrightarrow \mathbf{p}_{2}$. In the term $A_{\Gamma_{2}}\left(\mathbf{p}_{-} ; \tau\right)$ (7), the relative phase

$$
\Phi=\left(E-E_{g}\right) \tau+2 \phi_{12}
$$

is comprised of two contributions: $\left(E-E_{g}\right) \tau$, the difference in the phase accumulated during the temporal evolution of the two electronic wave packets produced by the pathways $\Gamma_{1}$ and $\Gamma_{2}$ due to the time delay $\tau$ between the two pulses; and $2 \phi_{12}=2\left(\phi_{1}-\phi_{2}\right)$, twice the relative CEP. Note that $E_{g}=-2.9037 \mathrm{au}$ is the energy of the $\left|{ }^{1} S_{0}^{e}\right\rangle$ ground state; $E=\left(p_{1}^{2}+p_{2}^{2}\right) / 2$ is the energy of the final $\left|\Phi_{\mathbf{p}_{1}, \mathbf{p}_{2}}^{(-)}\right\rangle$state. Moreover, the phase $\psi$ in the term $A_{\Gamma_{12}}\left(\mathbf{p}_{-} ; \tau\right)(8)$,

$$
\psi=\left(E \tau+\phi_{12}\right)
$$

shows that when the electron wave packet is created via the path $\Gamma_{12}$, the first electron wave packet (created via the path $\Gamma_{1}$ ) has already acquired a phase of $-E \tau$.

It is important to note that for our BTB detection geometry (see figure 1(b)), the PT amplitude (5) has the same form in either the basis of reduced two-electron momenta, $\mathbf{p}_{ \pm}$, the basis of conventional momenta, $\mathbf{p}_{1,2}$, or the basis of Jacobi momenta, $\mathbf{P}_{ \pm}$. The latter basis is defined respectively by the center-of-mass momentum, $\mathbf{P}_{+}=\mathbf{p}_{1}+\mathbf{p}_{2}$, and the relative momentum, $\mathbf{P}_{-}=\left(\mathbf{p}_{1}-\mathbf{p}_{2}\right) / 2$, of the ionized electron-pair [34-36]. Indeed, for the BTB geometry, $\mathbf{p}_{+}=0$, thus $\mathbf{p}=\hat{\mathbf{p}}_{1}=-\hat{\mathbf{p}}_{2}$ and $\mathbf{P}_{ \pm}$are all collinear since $\mathbf{P}_{+}=$ $\left(p_{1}-p_{2}\right) \mathbf{p}$ and $\mathbf{P}_{-}=\left[\left(p_{1}+p_{2}\right) / 2\right] \mathbf{p}$.

Using equations (4) and (5), the SDP for TPDI of He by a pair of oppositely circularly polarized pulses for our BTB detection geometry can be written as:

$$
\begin{aligned}
\mathcal{W}_{-\xi}^{\xi}\left(\mathbf{p}_{-} ; \tau\right)= & \left|A_{\Gamma_{1}}\left(\mathbf{p}_{-}\right)+A_{\Gamma_{2}}\left(\mathbf{p}_{-} ; \tau\right)\right|^{2}+\left|A_{\Gamma_{12}}\left(\mathbf{p}_{-} ; \tau\right)\right|^{2} \\
& +2 \operatorname{Re}\left[A_{\Gamma_{1}}^{*}\left(\mathbf{p}_{-}\right) A_{\Gamma_{12}}\left(\mathbf{p}_{-} ; \tau\right)\right] \\
& +2 \operatorname{Re}\left[A_{\Gamma_{2}}^{*}\left(\mathbf{p}_{-}\right) A_{\Gamma_{12}}\left(\mathbf{p}_{-} ; \tau\right)\right]
\end{aligned}
$$

which involves four terms. Substituting the geometric factor (9) in (6) and (7), the first term in the SDP (13), which describes Ramsey interference between the TPDI pathways $\Gamma_{1}$ and $\Gamma_{2}$, is given by:

$$
\begin{aligned}
& \left|A_{\Gamma_{1}}\left(\mathbf{p}_{-}\right)+A_{\Gamma_{2}}\left(\mathbf{p}_{-} ; \tau\right)\right|^{2}=\left|h_{-}(\rho)\right|^{2} \sin ^{4} \theta \\
& \quad \times \cos ^{2}\left[\left(E-E_{g}\right) \tau / 2+\phi_{12}-2 \xi \varphi\right],
\end{aligned}
$$

where $\xi=\xi_{1}=-\xi_{2}= \pm 1$ corresponds to a right-left ( + ) or a left-right (-) circularly-polarized pair of pulses. Equation (14) depends on the time delay $\tau$ via the Ramsey relative phase $\left(E-E_{g}\right) \tau$, and also on both the polar angle $\theta$ and azimuthal angle $\varphi$ of the Jacobi momentum $\mathbf{P}_{-} \propto \mathbf{p}_{-}$.

The second term in the $\operatorname{SDP}(13)$ is $\left|A_{\Gamma_{12}}\left(\mathbf{p}_{-} ; \tau\right)\right|^{2} \equiv$ $\left|A_{\Gamma_{12}}(\rho, \theta ; \tau)\right|^{2}$, which describes the pump-probe transition, does depend on the time delay $\tau$, but not on the azimuthal angle $\varphi$ of the electron pair. The third term in the SDP (13), 
which describes interference between the pathways $\Gamma_{1}$ and $\Gamma_{12}$, is

$$
\begin{aligned}
& 2 \operatorname{Re}\left[A_{\Gamma_{1}}^{*}\left(\mathbf{p}_{-}\right) A_{\Gamma_{12}}\left(\mathbf{p}_{-} ; \tau\right)\right]=2\left|h_{-}^{*} A_{\Gamma_{12}}\right| \sin ^{2} \theta \\
& \quad \times \cos ^{2}\left[\left(E \tau+\phi_{12}+\Theta(\rho, \theta, \tau)\right) / 2-\xi \varphi\right],
\end{aligned}
$$

while the fourth term in the SDP (13), which describes interference between the path $\Gamma_{2}$ and the path $\Gamma_{12}$, is

$$
\begin{aligned}
& 2 \operatorname{Re}\left[A_{\Gamma_{2}}^{*}\left(\mathbf{p}_{-}\right) A_{\Gamma_{12}}\left(\mathbf{p}_{-} ; \tau\right)\right]=-2\left|h_{-}^{*} A_{\Gamma_{12}}\right| \sin ^{2} \theta \\
& \quad \times \sin ^{2}\left[\left(E_{g} \tau-\phi_{12}+\Theta(\rho, \theta, \tau)\right) / 2+\xi \varphi\right] .
\end{aligned}
$$

The arguments $\rho$ for $h_{-}$and $(\rho, \theta ; \tau)$ for $A_{\Gamma_{12}}$ on the righthand side of equations (15) and (16) have been suppressed to simplify the notation. In equations (15) and (16), the $\phi_{12}$-independent dynamical phase, $\Theta(\rho, \theta, \tau)$, defined by

$$
\tan \Theta(\rho, \theta, \tau)=\frac{\operatorname{Im}\left[h_{-}^{*}(\rho) A_{\Gamma_{12}}(\rho, \theta, \tau)\right]}{\operatorname{Re}\left[h_{-}^{*}(\rho) A_{\Gamma_{12}}(\rho, \theta, \tau)\right]},
$$

depends on the time delay $\tau$, the energy $E$ and the polar angle $\theta$ of the electron pair.

Using the Hermitian stationary Green's function in the second-order PT amplitude, $A_{\Gamma_{12}}(\rho, \theta, \tau)$ can be approximated as a sum over intermediate autoionizing (doublyexcited) states (see figure 1(a)) in which we introduce the decay factors $\mathrm{e}^{-\tau / \tau_{k}}$ :

$$
A_{\Gamma_{12}}(\rho, \theta, \tau)=\sum_{k} \mathrm{e}^{-\mathrm{i} \epsilon_{k} \tau} A_{\Gamma_{12}}^{k}(\rho, \theta) \mathrm{e}^{-\tau / \tau_{k}},
$$

where $k$ runs from 1 to $N$, with $N$ being the number of doublyexcited states (with energy $\epsilon_{k}$ and lifetime $\tau_{k}$ ) lying within the bandwidth of the pump pulse. Using equation (18), the pumpprobe signal $\left|A_{\Gamma_{12}}(\rho, \theta, \tau)\right|^{2}$ becomes:

$$
\begin{aligned}
& \left|A_{\Gamma_{12}}(\rho, \theta, \tau)\right|^{2}=\sum_{k}\left|A_{\Gamma_{12}}^{k}(\rho, \theta)\right|^{2} \mathrm{e}^{-2 \tau / \tau_{k}} \\
& \quad+2 \sum_{k} \sum_{k^{\prime}>k}\left|A_{\Gamma_{12}}^{k *}(\rho, \theta)\right|\left|A_{\Gamma_{12}}^{k^{\prime}}(\rho, \theta)\right| \mathrm{e}^{-\tau / \tau_{k}} \mathrm{e}^{-\tau / \tau_{k^{\prime}}} \\
& \quad \times \cos \left[\left(\gamma_{k^{\prime}}-\gamma_{k}\right)+\left(\epsilon_{k}-\epsilon_{k^{\prime}}\right) \tau\right],
\end{aligned}
$$

where $\gamma_{k} \equiv \gamma_{k}(\rho, \theta)$ is the phase of the dynamical parameter $A_{\Gamma_{12}}^{k}(\rho, \theta)$; and the term $\left|h_{-}^{*}(\rho) A_{\Gamma_{12}}(\rho, \theta, \tau)\right|$ in equations (15) and (16) is given by:

$$
\begin{aligned}
& \left|h_{-}^{*}(\rho) A_{\Gamma_{12}}(\rho, \theta, \tau)\right|^{2}=\sum_{k}\left|h_{-}^{*}(\rho) A_{\Gamma_{12}}^{k}(\rho, \theta)\right|^{2} \mathrm{e}^{-2 \tau / \tau_{k}} \\
& \quad+2 \sum_{k} \sum_{k^{\prime}>k}\left|h_{-}(\rho) A_{\Gamma_{12}}^{k *}(\rho, \theta)\right| \\
& \quad \times\left|h_{-}^{*}(\rho) A_{\Gamma_{12}}^{k^{\prime}}(\rho, \theta)\right| \mathrm{e}^{-\tau / \tau_{k}} \mathrm{e}^{-\tau / \tau_{k^{\prime}}} \\
& \quad \times \cos \left[\left(\alpha_{k^{\prime}}-\alpha_{k}\right)+\left(\epsilon_{k}-\epsilon_{k^{\prime}}\right) \tau\right]
\end{aligned}
$$

where $\alpha_{k} \equiv \alpha_{k}(\rho, \theta)$ is the phase of the dynamical parameter $h_{-}^{*}(\rho) A_{\Gamma_{12}}^{k}(\rho, \theta)$. One sees that autoionization decays with rates $1 / \tau_{k}$ and QB-II processes with frequencies $\left(\epsilon_{k}-\epsilon_{k^{\prime}}\right)$ are present in equations (15) and (16) owing to the term $\left|h_{-}^{*}(\rho) A_{\Gamma_{12}}(\rho, \theta, \tau)\right|(20)$. They are also present in the pumpprobe term $\left|A_{\Gamma_{12}}(\rho, \theta ; \tau)\right|^{2}$ (19).

Any QB-I phenomena present in our TPDI process stem from the dynamical phase $\Theta(\rho, \theta, \tau)(17)$ in equations (15) and (16), which can be seen as follows. Doubly-excited states converging to the $\mathrm{He}^{+}(n=2,3)$ thresholds have lifetimes $\tau_{k}$ of several femtoseconds, which are very long compared to the few hundred attosecond time delays $\tau$ employed here. Thus, one may set $\mathrm{e}^{-\tau / \tau_{k}} \rightarrow 1$ in $A_{\Gamma_{12}}(\rho, \theta, \tau)(18)$. Among transitions from the ${ }^{1} \mathrm{~S}^{e}$ ground state to ${ }^{1} \mathrm{P}^{o}$ doubly-excited states involved in $A_{\Gamma_{12}}(\rho, \theta, \tau)(18)$, the $(2 s 2 p){ }^{1} \mathrm{P}^{o}$ doubly-excited state (with energy $\epsilon_{2 s 2 p}=-0.693 \mathrm{au}$ and lifetime $\tau_{2 s 2 p} \simeq 17.7 \mathrm{fs}$ ) has the largest oscillator strength. Indeed, its oscillator strength is about 6,15 , and 30 times larger than those for the $(2 s 3 p){ }^{1} \mathrm{P}^{o},(2 s 4 p){ }^{1} \mathrm{P}^{o}$, and $(2 s 5 p){ }^{1} \mathrm{P}^{o}$ doublyexcited states, respectively [37]. If we retain only the $(2 s 2 p){ }^{1} \mathrm{P}^{o}$ contribution in equation (18), then equation (17) may be expressed as

$$
\Theta(\rho, \theta, \tau)=\alpha_{2 s 2 p}(\rho, \theta)-\epsilon_{2 s 2 p} \tau,
$$

where $\alpha_{2 s 2 p}(\rho, \theta)$ is the phase of the dynamical parameter $h_{-}^{*}(\rho) A_{\Gamma_{12}}^{2 s 2 p}(\rho, \theta)$ describing the interference of path $\Gamma_{1}$ (or $\Gamma_{2}$ ) with path $\Gamma_{12}$. Substituting $\Theta(\rho, \theta, \tau)(21)$ in equation (16), one sees that the interference term $2 \operatorname{Re}\left[A_{\Gamma_{2}}^{*}\left(\mathbf{p}_{-}\right) A_{\Gamma_{12}}\left(\mathbf{p}_{-} ; \tau\right)\right]$ between the paths $\Gamma_{2}$ and $\Gamma_{12}$ exhibits QB-I with a period $T_{2 s 2 p}=2 \pi /\left(\epsilon_{2 s 2 p}-E_{g}\right) \simeq 69$ as.

\section{Numerical results and analysis for TPDI of He via doubly-excited states}

Our goal in presenting our numerical results is to demonstrate how doubly-excited state dynamics can affect the two-electron momentum, angular, and energy distributions in TPDI of He by two time-delayed, oppositely circularly-polarized attosecond pulses. This is done in two ways. First, we compare results for attosecond pulses whose carrier frequency $\omega$ is either on resonance $(\omega=60 \mathrm{eV}$, see figure 1(a)) or off resonance $(\omega=45 \mathrm{eV})$ with the transition from the $\mathrm{He}$ ground state to the $(2 s 2 p){ }^{1} \mathrm{P}^{o}$ doubly-excited state. In each case our pulses have $n=6$ optical cycles, cosine-squared envelopes, and peak intensities of $10^{14} \mathrm{~W} \mathrm{~cm}^{-2}$. The pulse bandwidths and durations in the resonant case are respectively $\Delta \omega \simeq 1.44 \omega / n=14.4 \mathrm{eV}$ and $T_{r}=413.6$ as, while those in the nonresonant case are $\Delta \omega=10.8 \mathrm{eV}$ and $T_{\mathrm{nr}}=551.4$ as. This comparison is particularly dramatic for the two-electron momentum distributions: the vortex pattern of the distributions is completely changed when the carrier frequency is on resonance with the $(2 s 2 p){ }^{1} \mathrm{P}^{o}$ state. Second, for pulses having a carrier frequency $\omega=60 \mathrm{eV}$ that is on resonance with the $(2 s 2 p){ }^{1} \mathrm{P}^{o}$ doubly-excited state, we compare our complete results with results in which we exclude final state orbital angular momenta that are only possible for the TPDI pathway $\Gamma_{12}$ corresponding to one-photon absorption from each pulse (see figure 1(a)). This comparison is particularly dramatic for the two-electron momentum, angular, and energy distributions, whose characters are completely changed by transitions corresponding to the resonant $\Gamma_{12}$ pathway.

In the results presented below, our focus for the energy spectrum of the ionized pair of electrons is on the range $10 \leqslant E \leqslant 70 \mathrm{eV}$ for the resonant transition and $0 \leqslant E \leqslant$ 

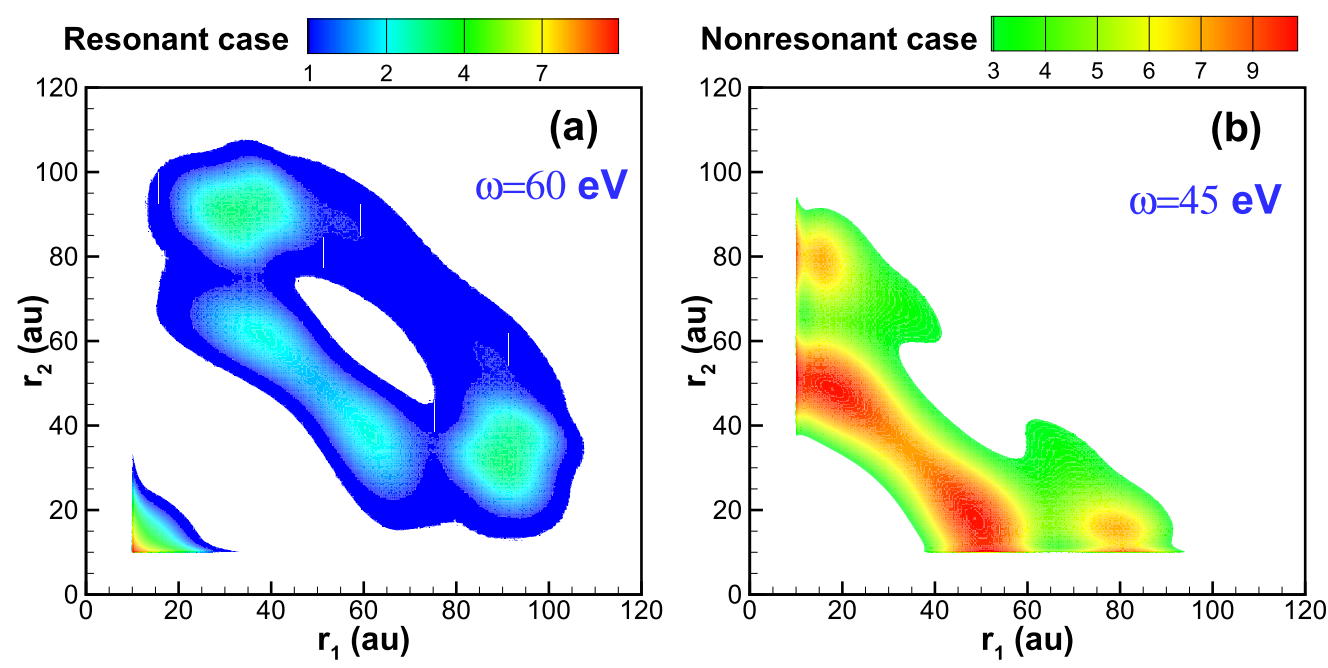

Figure 2. Radial probability distribution of the two-electron wave packet, $\Psi\left(\mathbf{r}_{1}, \mathbf{r}_{2}, t\right)$ (with the ground-state, singly-excited states, and singlecontinuum components subtracted), integrated over the angular variables $\left(\hat{\mathbf{r}}_{1}, \hat{\mathbf{r}}_{2}\right)$ at a time $T_{p}=30$ au after the end of the pair of nonoverlapping, right-left circularly-polarized, attosecond pulses, each having an intensity of $10^{14} \mathrm{~W} \mathrm{~cm}^{-2}$. (a) Results for pulses having the resonant carrier frequency, $\omega=60 \mathrm{eV}$, bandwidth $\Delta \omega \simeq 1.44 \omega / n=14.4 \mathrm{eV}$, where $n=6$ cycles, pulse duration $T_{r}=413.6$ as, and time delay $\tau=T_{r}$. (b) Results for pulses having the nonresonant carrier frequency, $\omega=45 \mathrm{eV}$, bandwidth $\Delta \omega \simeq 10.8 \mathrm{eV}$, pulse duration $T_{\mathrm{nr}}=551.4$ as, and time delay $\tau=T_{\mathrm{nr}}$. Observe in (a) the significant probability around $\left(r_{1}, r_{2}\right)=10-30$ au corresponding to population of doubly-excited states by single photon transitions from the first pulse. See text for discussion.

$32.6 \mathrm{eV}$ for the nonresonant transition. In each case, the excess energy $E$ can be shared unequally or equally by the two electrons. Figures 2(a) and (b) show the radial probability distributions of the two-electron wave packet, $\Psi\left(\mathbf{r}_{1}, \mathbf{r}_{2}, t\right)$, integrated over the angular variables $\left(\hat{\mathbf{r}}_{1}, \hat{\mathbf{r}}_{2}\right)$ at the time $T_{p}=30$ au after the end of our pair of non-overlapping rightleft circularly-polarized attosecond pulses for the resonant and nonresonant carrier frequencies, respectively. In each case, the ground-state, singly-excited states, and single-continuum components have been subtracted. The signal present in figure 2(a) around $\left(r_{1}, r_{2}\right)=10-30 \mathrm{au}$ and absent in figure $2(\mathrm{~b})$ is the doubly-excited part of the radial probability distribution involving ${ }^{1} \mathrm{P}_{+1}^{o}$ doubly-excited states whose lifetimes range from 17.7 fs to $0.24 \mathrm{~ns}$ for those converging to the $\operatorname{He}^{+}(n=2)$ threshold, and from 3.4 fs to $0.86 \mathrm{ps}$ for those converging to the $\operatorname{He}^{+}(n=3)$ threshold [38]. The signals seen at large radii $r_{1}$ and $r_{2}$ in figures 2(a) and (b) are the doubly-ionized parts of the radial probability distribution.

\subsection{Doubly-excited state effects on the two-electron momentum distributions}

The distribution (3) in terms of the Jacobi momentum $\mathbf{P}_{-}$in the polarization plane for TPDI of He produced by a pair of right-left circularly-polarized attosecond pulses having the resonant carrier frequency $\omega=60 \mathrm{eV}$, CEP $\phi_{1}=\phi_{2}=0$, and duration $T_{r}=413.6$ as, is displayed in figure 3(a) for a time delay $\tau=T_{r}=413.6$ as and in figure 3(c) for a time delay $\tau=500$ as. Plotted in figure 3(d) is the corresponding distribution for the nonresonant case (i.e. for $\omega=45 \mathrm{eV}$, $\phi_{1}=\phi_{2}=0$, and duration $T_{\mathrm{nr}}=551.4$ as) for a time delay $\tau=T_{\mathrm{nr}}=551.4$ as. In both resonant and nonresonant cases, the two ionized electrons unequally share the excess energy $E$ in the proportion $17.5 \%: 82.5 \%$, the two pulses do not overlap, and the TDSE numerical results include both ${ }^{1} \mathrm{~S}^{e}$ and ${ }^{1} \mathrm{D}^{e}$ final continuum states with $M=0, \pm 2$.

In figure 3(d), one sees that the $\mathbf{P}_{-}$distribution in the polarization plane for the nonresonant transition exhibits a four-arm spiral vortex pattern with a counterclockwise handedness. Moreover, the vortex pattern has a fourfold rotational symmetry, i.e. since the four spiral arms are equally separated by $\pi / 2$. As the time delay $\tau$ increases, the spiral becomes wound more densely (not shown). Reversing the ordering of the pulse pair helicities leads to a vortex pattern with a clockwise handedness (not shown). As nonresonant transitions by non-overlapping pulses only involve the two ionization paths $\Gamma_{1}$ and $\Gamma_{2}$, these features of our TDSE results can be fully explained by PT formula (14). Specifically, in the polarization plane $(\theta=\pi / 2)$, the first term in the SDP (13), i.e. $\left|A_{\Gamma_{1}}\left(\mathbf{p}_{-}\right)+A_{\Gamma_{2}}\left(\mathbf{p}_{-} ; \tau\right)\right|^{2}(14)$, which involves only the two paths $\Gamma_{1}$ and $\Gamma_{2}$, has the form of a four-arm Archimedean spiral vortex pattern. Indeed, for our BTB emission geometry (see figure $1(\mathrm{a})), \beta=\cos ^{-1}\left(\hat{\mathbf{p}}_{1} \cdot \hat{\mathbf{p}}_{2}\right)=\pi$, so that the dynamical parameter $\left|h_{-}\left(p_{1}, p_{2}, \cos \beta\right)\right|^{2}$ in (14) has no angular dependence. Hence, the vortex pattern is defined by the zeros and the maxima of the kinematical factor on the right-hand side of (14). The zeros occur for $\left(E-E_{g}\right) \tau / 2+\phi_{12}-2 \xi \varphi=$ $(2 n+1) \pi / 2$ and the maxima occur for $\left(E-E_{g}\right) \tau / 2+\phi_{12}-$ $2 \xi \varphi=n \pi$, where $n=0, \pm 1, \pm 2 \ldots$, with $0 \leqslant \varphi \leqslant 2 \pi$. The dependences of the azimuthal angle $\varphi$ on the excess energy $E$ at these maximum and zero values of (14) are thus given by:

$$
\begin{aligned}
& \varphi_{n}^{\max }(E)=-\xi\left[\pi n-\left(2 \phi_{12}-\tau E_{g}\right) / 2-\tau E / 2\right] / 2 \\
& \varphi_{n}^{\text {zero }}(E)=-\xi\left[\pi / 2+\pi n-\left(2 \phi_{12}-\tau E_{g}\right) / 2-\tau E / 2\right] / 2
\end{aligned}
$$



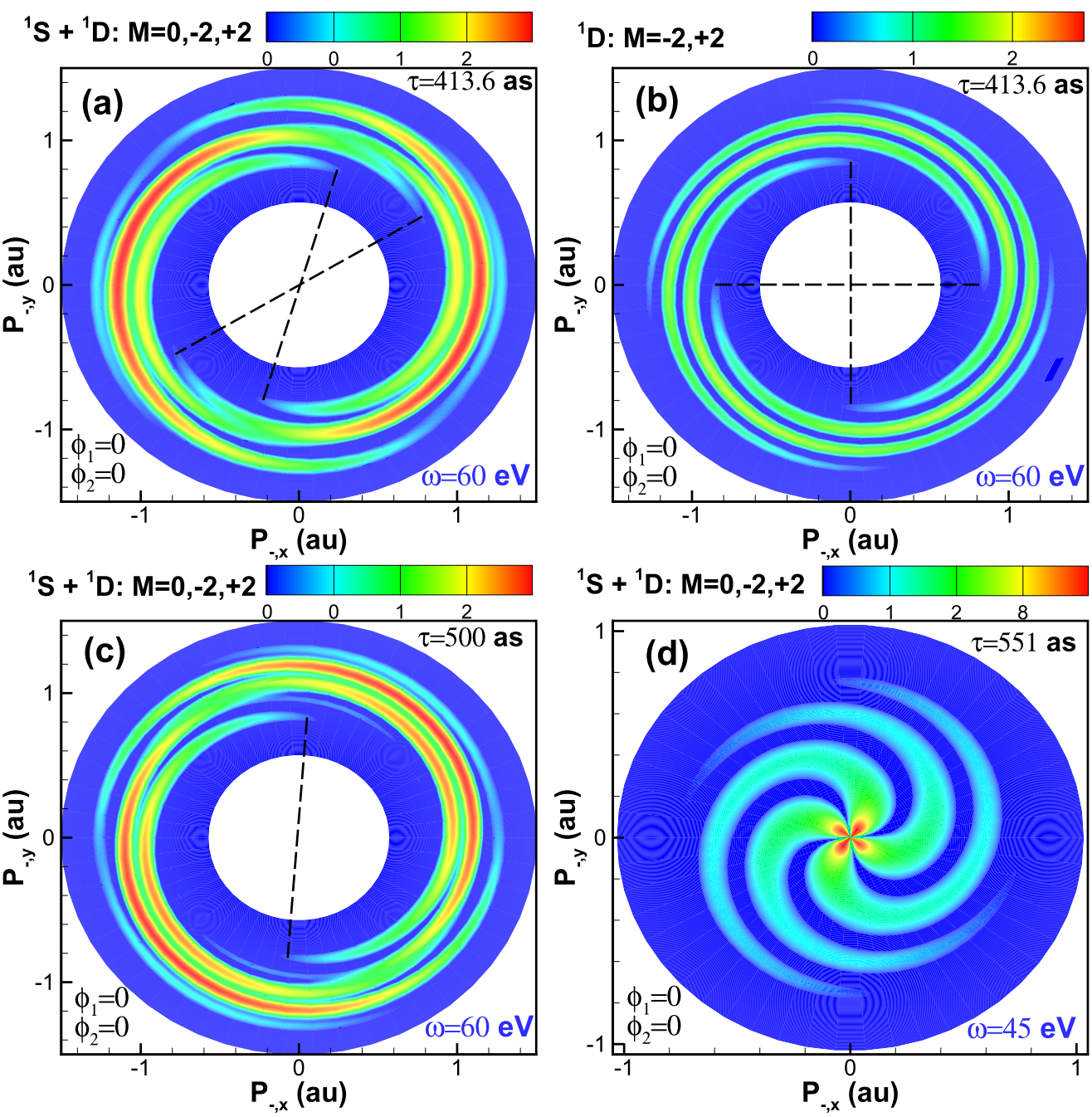

Figure 3. Two-electron momentum distributions (calculated using equation (3) and analyzed in the text using equations (13)-(16)) for TPDI of He produced by a pair of non-overlapping, right-left circularly-polarized, six-cycle, attosecond pulses. (a)-(c) The two pulses have the same resonant carrier frequency $\omega=60 \mathrm{eV}$, duration $T_{r}=413.6$ as, and are delayed in time by $\tau=T_{r}=413.6$ as $((\mathrm{a})$ and (b)) and $\tau=500$ as (c). (d) The two pulses have the nonresonant carrier frequency $\omega=45 \mathrm{eV}$, a duration $T_{\mathrm{nr}}=551.4$ as, and are delayed in time by $\tau=T_{\mathrm{nr}}=551.4$ as. In all panels, the two pulses have the same CEP $\phi_{1}=\phi_{2}=0$, a cosine-squared field envelope, and an intensity of $10^{14} \mathrm{~W} \mathrm{~cm}^{-2}$. The results in (a), (c) and (d) include all three ionization pathways (i.e. ${ }^{1} \mathrm{~S}^{e}$ and ${ }^{1} \mathrm{D}^{e}$ final states with $M=0, \pm 2$ ), while in (b) only the two direct ionization pathways (i.e. ${ }^{1} \mathrm{D}^{e}$ final states with $M= \pm 2$ ) are included. The two electrons are emitted BTB in the polarization plane and share the excess energy unequally in the proportion $17.5 \%: 82.5 \%$.

Equation (22) defines Archimedean spirals (or helixes) in the $(E, \varphi)$ plane. As $\varphi_{n}^{\max }(E)$ and $\varphi_{n}^{\text {zero }}(E)$, shifted by the angle $\pi / 4$ with respect to each other, vary with energy $E$ (through possibly many $2 \pi$ cycles, depending upon $\tau$ ), they trace out the maxima and the zeros of the term (14). Since $|\xi|=1$, the pattern is a four-arm helical spiral, corresponding to $n=0,1,2,3$; other values of $n$ replicate the same lines.

In contrast to the nonresonant case, in the resonant case in which doubly-excited states are populated by one-photon transitions from the first pulse (see figures 1(a) and 2(a)), the structure of the $\mathbf{P}_{-}$distribution in the polarization plane changes dramatically, as shown in figure 3 (a) for $\omega=60 \mathrm{eV}$ and $\tau=T_{r}=413.6$ as. Even though the four arms of the spiral in figure 3(a) are still visible, its fourfold rotational symmetry is broken, as illustrated by the two black dashed lines in figure 3(a) that connect the onsets of opposite pairs of the four spiral arms. As shown in figure 3(c), further delaying the second pulse by only 86.4 as leads to overlap of the spiral arms so that they almost appear as a two-arm spiral pattern (see the single black dashed line in figure 3(c)). Despite the breakdown of the fourfold rotational symmetry of the vortex pattern as the time delay $\tau$ increases, the handedness of the spiral is preserved.

In order to understand the difference between the momentum distributions for the nonresonant (figure 3(d)) and the resonant (figures 3(a) and (c)) cases, we have carried out a TDSE calculation for the $\mathbf{P}_{-}$distribution (3) that includes only the ${ }^{1} \mathrm{D}^{e}$ final continuum states with $M= \pm 2$. The result is shown in figure 3(b) for $\omega=60 \mathrm{eV}$ and $\tau=T_{r}=413.6$ as. Just as for the momentum distribution for the nonresonant transition in figure 3(d), one sees that the momentum distribution for the $M= \pm 2$ final state channels has a four-start 
Table 1. Angular shift, $\Delta \varphi\left(E=2 \omega+E_{g}, \tau\right)$, of the angular distributions in figure 4 with respect to the horizontal axis as a function of time delay $\tau$.

\begin{tabular}{ccccccccc}
\hline$\tau$ (as) & 413.6 & 424 & 434 & 444 & 448.1 & 454 & 464 & 482.6 \\
\hline$\Delta \varphi(E, \tau)$ & $0^{\circ}$ & $27.2^{\circ}$ & $53.3^{\circ}$ & $79.4^{\circ}$ & $90^{\circ}$ & $105.4^{\circ}$ & $131.6^{\circ}$ & $180^{\circ}$ \\
\hline
\end{tabular}

spiral vortex structure and exhibits fourfold rotational symmetry since the onsets of the four spiral arms are equally separated by $90^{\circ}$ (see the two black dashed lines in figure 3(b)). This result holds for time delays $\tau>T_{r}$, as may be understood from the PT formula (14). Hence, the difference between the nonresonant (figure 3(d)) and resonant (figures 3(a) and (c)) momentum distributions is due to the ionization channels that lead to ${ }^{1} \mathrm{~S}^{e}$ and ${ }^{1} \mathrm{D}^{e}$ final continuum states with $M=0$; i.e. it is due to the ionization path $\Gamma_{12}$ in the PT picture.

According to the PT equation (13) for the SDP, the amplitude $A_{\Gamma_{12}}$ for the ionization path $\Gamma_{12}$ enters its last three terms: the pump-probe term $\left|A_{\Gamma_{12}}(\rho, \theta ; \tau)\right|^{2}$, the interference term (15) between the pathways $\Gamma_{1}$ and $\Gamma_{12}$, and the interference term (16) between the pathways $\Gamma_{2}$ and $\Gamma_{12}$. As the pump-probe term $\left|A_{\Gamma_{12}}(\rho, \theta ; \tau)\right|^{2}$ is independent of the azimuthal angle $\varphi$ of the momentum $\mathbf{P}_{-}$, the breakdown of the fourfold rotational symmetry of the vortex patterns in figures 3(a) and (c) can only be due to the two interference terms (15) and (16), which differ in their kinematical structures. The kinematic factor $\cos ^{2}\left[\left(E \tau+\phi_{12}+\Theta(\rho, \theta, \tau)\right) / 2-\xi \varphi\right]$ of the interference term $2 \operatorname{Re}\left[A_{\Gamma_{1}}^{*}\left(\mathbf{p}_{-}\right) A_{\Gamma_{12}}\left(\mathbf{p}_{-} ; \tau\right)\right]$ (15) leads to two-arm spiral vortex patterns with the same handedness as the four-arm spirals produced by the term $\left|A_{\Gamma_{1}}\left(\mathbf{p}_{-}\right)+A_{\Gamma_{2}}\left(\mathbf{p}_{-} ; \tau\right)\right|^{2}$ (14). However, the helix equations for this two-arm spiral,

$\varphi_{n}^{\max }(E)=-\xi\left\{\pi n-\left[\phi_{12}+\Theta(\rho, \theta, \tau)\right] / 2-\tau E / 2\right\}$,

$\varphi_{n}^{\text {zero }}(E)=-\xi\left\{\pi / 2+\pi n-\left[\phi_{12}+\Theta(\rho, \theta, \tau)\right] / 2-\tau E / 2\right\}$

are modified by the dynamical phase $\Theta(\rho, \theta, \tau)$ (17). Correspondingly, the interference term $2 \operatorname{Re}\left[A_{\Gamma_{2}}^{*}\left(\mathbf{p}_{-}\right) A_{\Gamma_{12}}\left(\mathbf{p}_{-} ; \tau\right)\right](16)$ has a dipole-like kinematical factor $\sin ^{2}\left[\left(E_{g} \tau-\phi_{12}+\Theta\right.\right.$ $(\rho, \theta, \tau)) / 2+\xi \varphi]$ that depends on the excess energy $E$ only via the dynamical phase $\Theta(\rho, \theta, \tau)$ (17); hence it does not support a vortex pattern in the momentum distribution. The breakdown of the fourfold rotational symmetry of the vortex patterns thus stems only from the interference term $2 \operatorname{Re}\left[A_{\Gamma_{1}}^{*}\left(\mathbf{p}_{-}\right) A_{\Gamma_{12}}\left(\mathbf{p}_{-} ; \tau\right)\right](15)$.

\subsection{Doubly-excited state effects on the fixed-energy two- electron angular distributions}

For the same BTB detection geometry, energy-sharing configuration, and resonant pulse parameters used in section 4.1, we show in figure 4 the angular distributions in the polarization plane for a fixed excess energy $E=2 \omega+E_{g} \simeq 41 \mathrm{eV}$ and five time delays: $\tau=T_{r}=413.6$ as (figure 4(a)); $\tau=424$ as (figure 4(b)); $\tau=T_{r}+T_{2 s 2 p} / 2 \simeq 448.1$ as (figure 4(c)); $\tau=464$ as (figure $4(\mathrm{~d})$ ); and $\tau=T_{r}+T_{2 s 2 p} \simeq 482.6$ as (figure 4(e)), where $T_{2 s 2 p}=2 \pi /\left(\epsilon_{2 s 2 p}-E_{g}\right) \simeq 69$ as. In each panel of figure 4 , we compare TDSE results for (i) ${ }^{1} \mathrm{~S}_{0}^{e}$ and ${ }^{1} \mathrm{D}_{0, \pm 2}^{e}$ final states with those for (ii) ${ }^{1} \mathrm{D}_{ \pm 2}^{e}$ final states.

For a fixed time delay $\tau$, one sees in figure 4 that the angular distributions (ii) have a fourfold symmetric quadrupolelike shape, while those for (i) have a twofold symmetric quadrupole-like shape (since they exhibit two large lobes perpendicular to two small lobes). As the time delay $\tau$ increases, both angular distributions (i) and (ii) rotate counterclockwise with the same angular shift $\Delta \varphi\left(E=2 \omega+E_{g}, \tau\right)$ with respect to the horizontal axis; see table 1. From table 1 and figure 4, we see that the angular distributions (i) have a periodicity with increasing time delay $\tau$ of $2 \pi / \omega \simeq 69$ as, while the angular distributions (ii) have a periodicity of $\pi / \omega \simeq 34.5$ as.

To understand the TDSE results (i) and (ii), we use PT. The angular distribution (ii) is described by the first term (14) in the $\operatorname{SDP}(13)$. For $E=2 \omega+E_{g}$, the term (14) is $\left|A_{\Gamma_{1}}\left(\mathbf{p}_{-}\right)+A_{\Gamma_{2}}\left(\mathbf{p}_{-} ; \tau\right)\right|^{2} \propto\left|h_{-}(\rho)\right|^{2} \cos ^{2}\left(\omega \tau+\phi_{12}-2 \xi \varphi\right)$, which has a fourfold symmetric quadrupole-like shape and a periodicity of $\pi / \omega$ with increasing time delay $\tau$. Thus, the angular distribution (ii) is predicted to be unchanged for time delays $\tau_{n}=n \pi / \omega$, where $n \geqslant 12$ (for non-overlapping pulses) is an integer and where $\tau_{12}=T_{r}$. This is indeed the case as shown by comparing our TDSE angular distribution (ii) for $\tau=\tau_{12}=T_{r}$ in figure 4(a) with those for $\tau=\tau_{13}=T_{r}+$ $\pi / \omega$ in figure 4(c) and $\tau=\tau_{14}=T_{r}+2 \pi / \omega$ in figure 4(e). Using the helix equations (22), the angular shifts $\Delta \varphi(E, \tau)$ with $\tau$, given in table 1 , of the maxima of the angular distributions (ii) in figure 4 (relative to their positions in figure $4(\mathrm{a}))$ are predicted by PT to be $\Delta \varphi(E, \tau)=\omega \tau / 2$ modulo $2 \pi$.

The angular distribution (i) is described in PT by the SDP (13). Thus, its shape for a fixed time delay $\tau$ (i.e. its dependence on $\varphi$ ) is governed by the interplay between the first term (14) in (13), discussed above, with the two interference terms (15) and (16), since the second term in (13), $\left|A_{\Gamma_{12}}(\rho, \theta ; \tau)\right|^{2}$, is independent of $\varphi$. If we assume that the $(2 s 2 p){ }^{1} \mathrm{P}^{o}$ doubly-excited state is the dominant intermediate state in the ionization path $\Gamma_{12}$, we can use the expression (21) for the dynamical angle $\Theta(\rho, \theta, \tau)$ and approximate the QB-I frequency as $\left(\epsilon_{2 s 2 p}-E_{g}\right) \simeq \omega$. For $E=2 \omega+E_{g}$, the two interference terms (15) and (16) simplify as follows: $2 \operatorname{Re}\left[A_{\Gamma_{1}}^{*}\left(\mathbf{p}_{-}\right) A_{\Gamma_{12}}\right.$ $\left.\left(\mathbf{p}_{-} ; \tau\right)\right] \propto\left|h_{-}^{*}(\rho) A_{\Gamma_{12}}(\rho, \theta, \tau)\right| \cos ^{2}\left\{\left[\omega \tau+\phi_{12}+\alpha_{2 s 2 p} \quad(\rho\right.\right.$, $\theta)] / 2-\xi \varphi\}$ and $2 \operatorname{Re}\left[A_{\Gamma_{2}}^{*}\left(\mathbf{p}_{-}\right) A_{\Gamma_{12}}\left(\mathbf{p}_{-} ; \tau\right)\right] \propto-\mid h_{-}^{*}(\rho)$ $A_{\Gamma_{12}}(\rho, \theta, \tau) \mid \sin ^{2}\left\{\left[\omega \tau+\phi_{12}-\alpha_{2 s 2 p}(\rho, \theta)\right] / 2-\xi \varphi\right\}$. Both exhibit a dipolar shape and both have a period $T_{2 s 2 p}=2 \pi / \omega$, where $\omega \simeq\left(\epsilon_{2 s 2 p}-E_{g}\right)$ is the beat frequency between the 


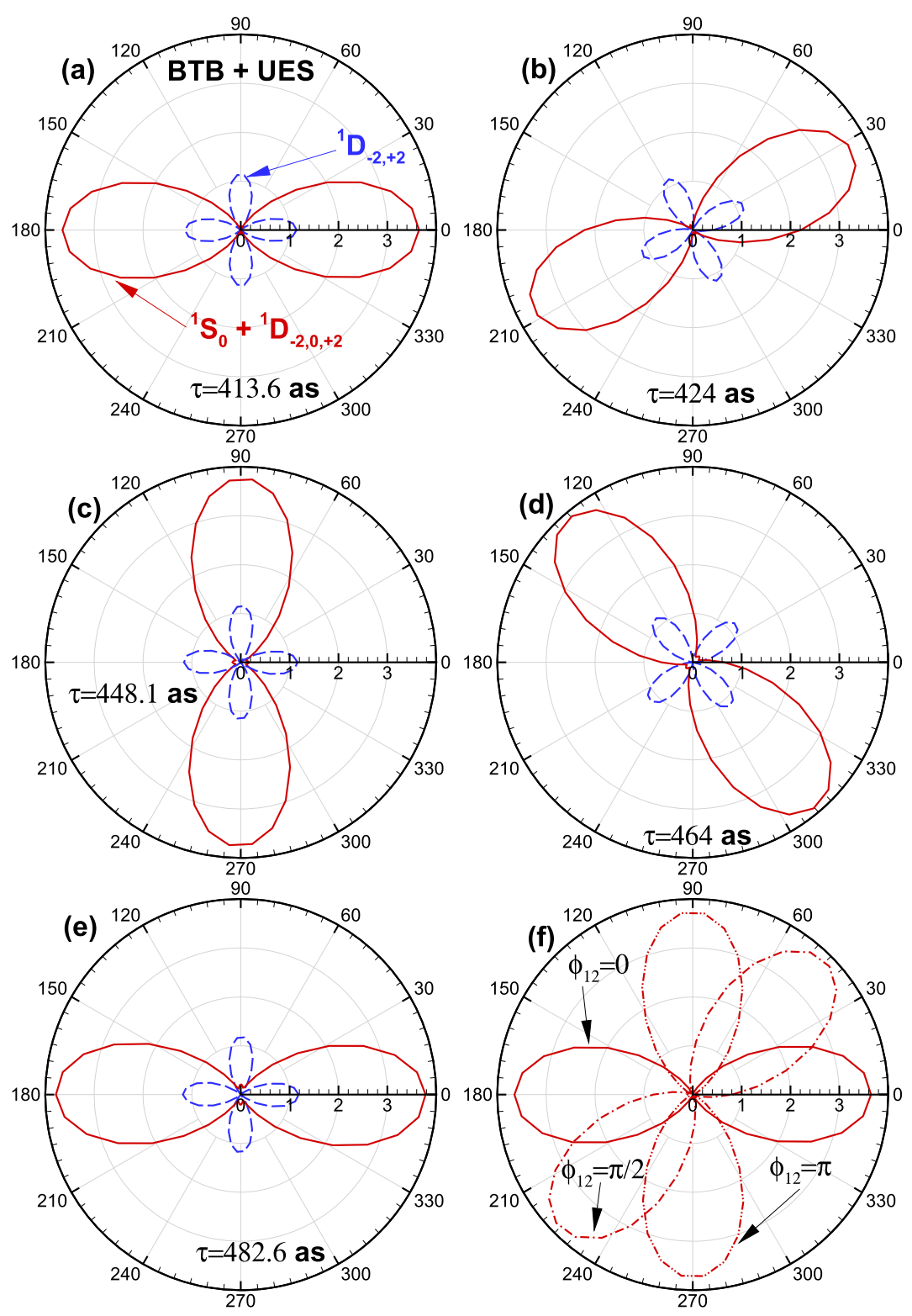

Figure 4. Angular distributions (in units of $10^{-7} \mathrm{au}$ ) for a fixed excess energy $E=2 \omega+E_{g} \simeq 41 \mathrm{eV}$ produced by a pair of non-overlapping, right-left circularly-polarized attosecond pulses having a relative $\operatorname{CEP} \phi_{12}=0$ for five values of the time delay $\tau$ : (a) $\tau=T_{r}=413.6$ as; (b) $\tau=424$ as; (c) $\tau=T_{r}+T_{2 s 2 p} / 2 \simeq 448.1$ as; (d) $\tau=464$ as; and (e) $\tau=T_{r}+T_{2 s 2 p} \simeq 482.6$ as, where $T_{2 s 2 p}=2 \pi / \omega \simeq 69$ as. (a)-(e) The solid (red) curves are our complete TDSE results (i) including all paths $\Gamma_{1}, \Gamma_{2}$, and $\Gamma_{12}$, which lead to final states ${ }^{1} \mathrm{~S}^{e}$ and ${ }^{1} \mathrm{D}^{e}$ with $M=0$, \pm 2 , whereas the dashed (blue) curves are our TDSE results (ii) including only the paths $\Gamma_{1}$ and $\Gamma_{2}$, which lead to final states ${ }^{1} \mathrm{D}^{e}$ with $M= \pm 2$. (f) The $\phi_{12}$-sensitivity of the angular distribution (i). Our TDSE results are calculated using equation (3) and are analyzed in the text using equations (13)(16). Each pulse has a $\cos ^{2}$ profile, a carrier frequency $\omega=60 \mathrm{eV}$, an intensity $I=10^{14} \mathrm{~W} \mathrm{~cm}^{-2}, n=6$ cycles, and a duration $T_{r}=413.6$ as. The two electrons are emitted BTB in the polarization plane with a fixed energy sharing, $\varepsilon=E_{1} / E=1-E_{2} / E=17.5 \%$.

ground state and the $(2 s 2 p){ }^{1} \mathrm{P}^{o}$ doubly-excited state. Our TDSE results show that the magnitude of the dynamical amplitude $\left|h_{-}(\rho)\right|^{2}$, which is proportional to the transition probabilities for the paths $\Gamma_{1}$ and $\Gamma_{2}$, is comparable to the magnitude of the dynamical amplitude $\left|h_{-}^{*}(\rho) A_{\Gamma_{12}}(\rho, \theta, \tau)\right|$ in the interference terms involving either the path $\Gamma_{1}$ or the path $\Gamma_{2}$ with the path $\Gamma_{12}$. The magnitudes of the dynamical parameters $\Gamma_{j}^{L, M}$ for each final state channel $L, M$ and each pathway $j=1,2$, and 12 (which are extracted from our TDSE results, as described in the caption) are presented in figure 5. One sees that all dynamical amplitudes are of comparable magnitudes, indicating the possibility of significant constructive and destructive interference effects.

The effects of constructive and destructive interference of the terms in (13) on the angular distributions (i) in figure 4 are evident. The interplay between the term $\left|A_{\Gamma_{1}}\left(\mathbf{p}_{-}\right)+A_{\Gamma_{2}}\left(\mathbf{p}_{-} ; \tau\right)\right|^{2}$ (14) and the interference term $2 \operatorname{Re}\left[A_{\Gamma_{1}}^{*}\left(\mathbf{p}_{-}\right) A_{\Gamma_{12}}\left(\mathbf{p}_{-} ; \tau\right)\right](15)$ leads to two large lobes in the angular distribution (i) in one direction, whereas the interplay between this term (14) and the interference term $2 \operatorname{Re}\left[A_{\Gamma_{2}}^{*}\left(\mathbf{p}_{-}\right) A_{\Gamma_{12}}\left(\mathbf{p}_{-} ; \tau\right)\right]$ (16) leads to two small lobes in the angular distribution (i) in a perpendicular 


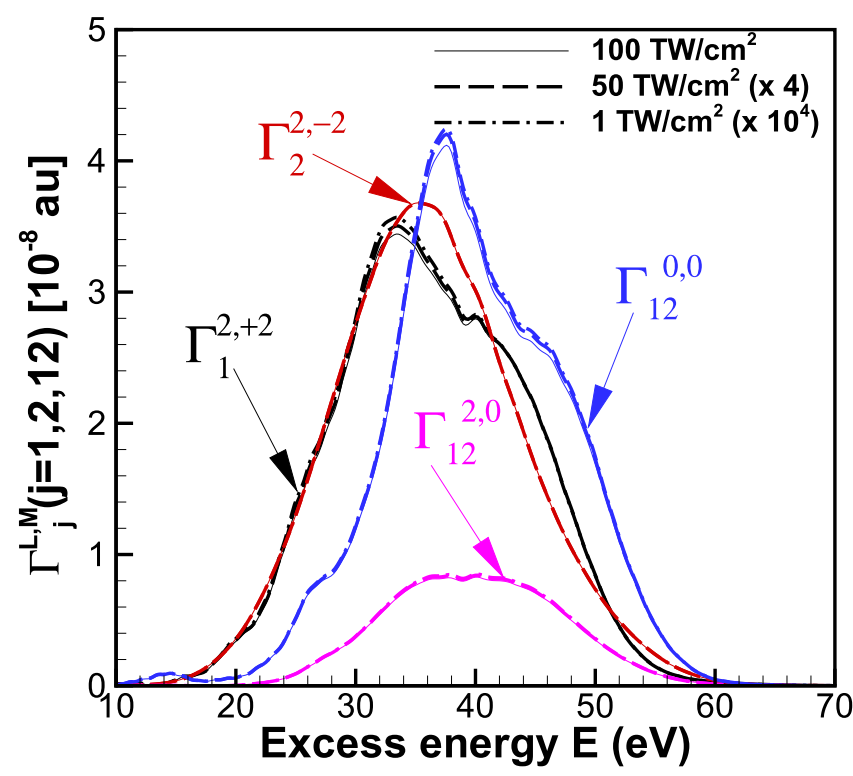

Figure 5. Dynamical parameters $\Gamma_{j}^{L, M}$ (where $j=1,2,12$ ) describing the ionization pathways $\Gamma_{1}, \Gamma_{2}$, and $\Gamma_{12}$, respectively. Theses parameters are extracted from TDSE calculations by including in the projection (3) only final states with $L=2, \quad M=0$ or $L=0, M=0$ for $j=12 ; L=2, M=+2$ for $j=1$; and $L=2, M=-2$ for $j=2$. Results for three laser pulse intensities are given: $10^{14} \mathrm{~W} \mathrm{~cm}^{-2}$ (thin solid lines), unscaled; $5 \times 10^{13}$ $\mathrm{W} \mathrm{cm}{ }^{-2}$ (dashed lines), scaled by a factor 4 ; and $10^{12} \mathrm{~W} \mathrm{~cm}^{-2}$ (dashdotted lines), scaled by a factor $10^{4}$. The results are for the BTB detection geometry and the pulse parameters are the same as in figure 4 .

direction. Hence, as $\tau$ varies, the resulting twofold symmetric quadrupole-like shape of the angular distribution (i) is expected to rotate counterclockwise with a frequency $\omega$; to have a periodicity (i.e. to be unchanged) when $\tau=n T_{2 s 2 p}=n(2 \pi / \omega)$, where $n$ is an integer; and to have one of its two large lobes located at $\omega \tau / 2$. These predictions for the shape, $\tau$-periodicity, and location of maxima are clearly shown in both figure 4 and table 1 , where the angular distributions (i) for $\tau=T_{r}=413.6$ as (see figure 4(a)) and $\tau=T_{r}+T_{2 s 2 p} \simeq 482.6$ as (see figure 4(d)) almost coincide. This $\tau$-periodicity of the angular distribution (i) at a fixed excess energy $E=2 \omega+E_{g} \simeq 41 \mathrm{eV}$ demonstrates the quantum beat between the ground state and the $(2 s 2 p){ }^{1} \mathrm{P}^{o}$ doublyexcited state.

Figure 4(f) shows the sensitivity of the angular distribution in the polarization plane to the relative CEP $\phi_{12}$, which may be understood by examination of the PT equations (14)-(16) for each of the terms in the SDP (13). One sees that each term in the trigonometric function has a phase proportional to $\phi_{12}-2 \xi \varphi$, indicating that the effect of the relative CEP $\phi_{12}$ on the $\varphi$-dependence of each term in the SDP (13) is the same. Hence, a change of the relative $\mathrm{CEP} \phi_{12}$ results in a global rotation of the angular distribution and also of the vortex pattern (not shown) by an angle $\phi_{12} / 2$.
Finally, we comment on how the angular distributions change when the fixed energy $E$ is shifted by an amount $\pm \delta$ from the resonance energy, i.e. when $E=2 \omega+E_{g} \pm \delta$. Note first that from figure 5 , the dynamical parameters $\Gamma_{j}^{L, M}$ have significant magnitude over a broad range of energy $E$. As $\tau$ varies, PT formula (14) predicts that the angular distributions (ii) exhibit a fourfold symmetric quadrupole-like shape with a periodicity $\tau_{n}=n \pi /(\omega \pm \delta / 2)$, where $n$ is an integer. In contrast, the angular distributions (i) always exhibit a twofold symmetric quadrupole-like shape with the interference term (15) having a rotation frequency of $\omega \pm \delta$, while the interference term (16) retains its same QB-I rotation frequency of $\omega \simeq\left(\epsilon_{2 s 2 p}-E_{g}\right)$. However, in both interference terms (15) and (16), the dynamical parameter $\alpha_{2 s 2 p}(\rho, \theta)$ is affected by the energy shift $\delta$. Despite these complications, both angular distributions (i) and (ii) for $E=2 \omega+E_{g} \pm \delta$ always have the same angular shift $\Delta \varphi(E, \tau)=(\omega \pm \delta / 2) \tau / 2$. All of these PT predictions have been confirmed by our TDSE results (not shown) for the angular distributions (i) and (ii) for four values of the excess energy $E$ : 34, 36, 38 and $40 \mathrm{eV}$.

\subsection{Doubly-excited state effects on the energy-integrated angular distributions}

The energy-integrated angular distribution for the SDP (13) takes the following form:

$$
\begin{aligned}
\mathcal{W}_{-\xi}^{\xi}(\theta, \varphi, \tau)= & \mathcal{W}_{\Gamma_{2}}^{\Gamma_{1}}(\theta)+\mathcal{W}_{\Gamma_{12}}^{\Gamma_{12}}(\theta, \tau)+\Upsilon_{\tau}^{\theta} \cos \left(\phi_{12}-2 \xi \varphi\right) \\
& -\Pi_{\tau}^{\theta} \sin \left(\phi_{12}-2 \xi \varphi\right) \\
= & \mathcal{W}_{\Gamma_{2}}^{\Gamma_{1}}(\theta)+\mathcal{W}_{\Gamma_{12}}^{\Gamma_{12}}(\theta, \tau)+\Lambda(\theta, \tau) \\
& \times \cos \left[\chi(\theta, \tau)+\phi_{12}-2 \xi \varphi\right] .
\end{aligned}
$$

The first term,

$$
\mathcal{W}_{\Gamma_{2}}^{\Gamma_{1}}(\theta) \simeq \frac{1}{2} \sin ^{4} \theta \int \mathrm{d} E\left|h_{-}(\rho)\right|^{2}
$$

stems from the term $\left|A_{\Gamma_{1}}\left(\mathbf{p}_{-}\right)+A_{\Gamma_{2}}\left(\mathbf{p}_{-} ; \tau\right)\right|^{2}$ (14) of the SDP (13) integrated over the energy $E$ under the assumption that $\left|h_{-}(\rho)\right|^{2}$ varies slowly compared to the trigonometric factor $\cos \left[\left(E-E_{g}\right) \tau+2 \phi_{12}-4 \xi \varphi\right]$. The second term,

$$
\mathcal{W}_{\Gamma_{12}}^{\Gamma_{12}}(\theta, \tau)=\int \mathrm{d} E\left|A_{\Gamma_{12}}(\rho, \theta ; \tau)\right|^{2},
$$

is the pump-probe term integrated over the energy $E$, which is independent of $\varphi$ but dependent on $\tau$. The third term of equation (24) describes interference between the $\Gamma_{12}$ and $\Gamma_{2}$ paths, where the dynamical amplitude $\Lambda(\theta, \tau)$ and angle $\chi(\theta, \tau)$ are defined by:

$$
\begin{gathered}
\Lambda(\theta, \tau)=\sin ^{2} \theta \sqrt{\Upsilon^{2}(\theta, \tau)+\Pi^{2}(\theta, \tau)}, \\
\tan \chi(\theta, \tau)=\Pi(\theta, \tau) / \Upsilon(\theta, \tau),
\end{gathered}
$$



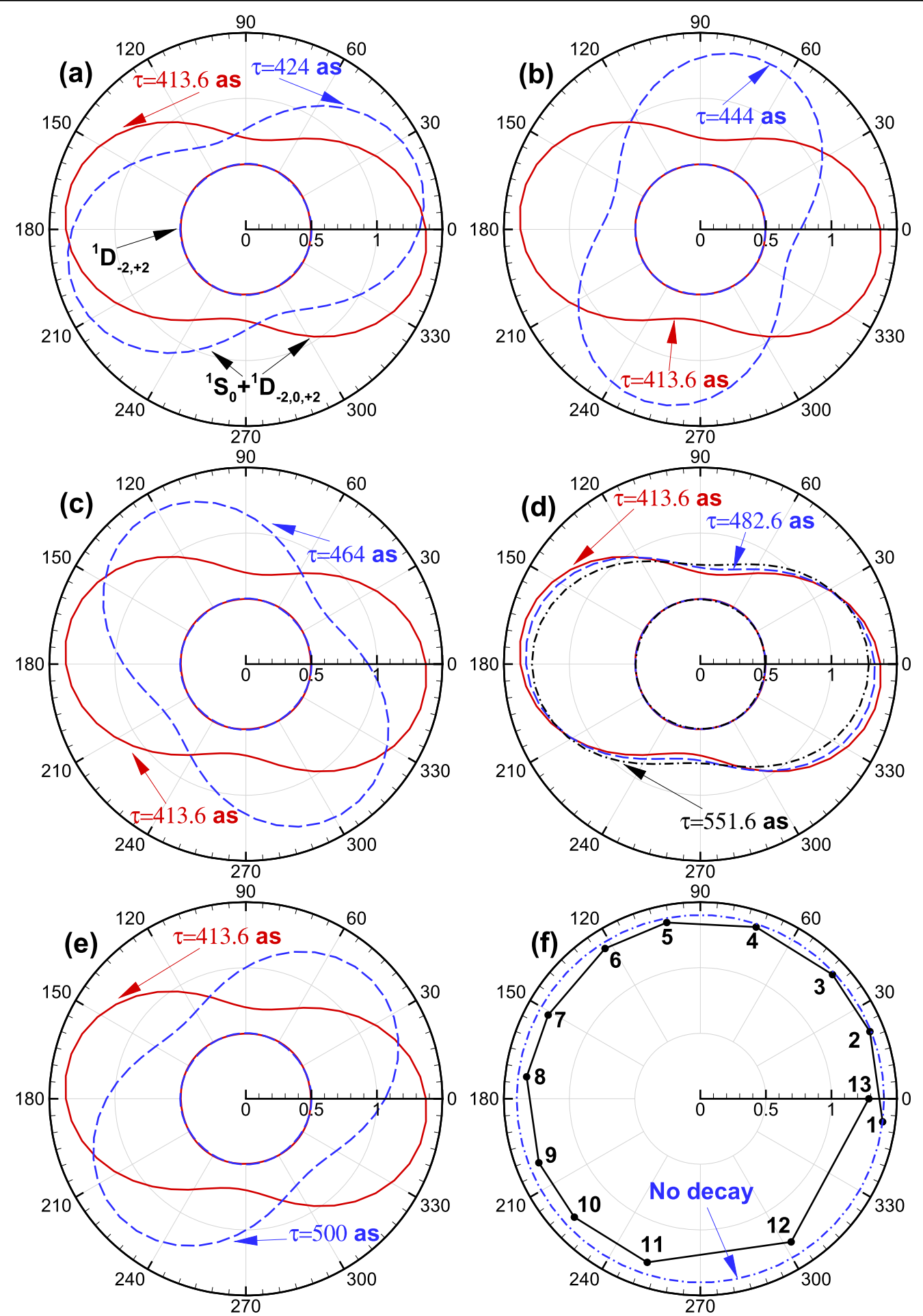

Figure 6. TDSE results for the energy-integrated angular distributions (i) and (ii) (in units of $10^{-7}$ au) produced by right-left circularly polarized attosecond pulses for seven values of the time-delay $\tau$, as indicated by the arrows in panels (a)-(e). The results (i) include all final states ${ }^{1} \mathrm{~S}_{0}^{e}$ and ${ }^{1} \mathrm{D}_{-2,0,+2}^{e}$ and have peanut shapes, while the results (ii), which are independent of $\tau$, include only ${ }^{1} \mathrm{D}_{ \pm 2}^{e}$ final states and have circular shapes. In each panel (a)-(e), TDSE results (i) for $\tau=T_{r}=413.6$ as (solid (red) curves) are shown for reference. Note that in panel (d) the TDSE (i) results for $\tau=T_{r}+n T_{2 s 2 p}$, where $n=0,1$, 2, (i.e. for 413.6, 482.6, and 551.6 as) nearly coincide, demonstrating a quantum beat periodicity of $T_{2 s 2 p}=2 \pi /\left(\epsilon_{2 s 2 p}-E_{g}\right) \simeq 69$ as. Panel (f) gives the maxima of the peanut-shaped angular distributions in (a)(e) for 13 times delays $\tau$ : (1) 413.6 as, (2) 424 as, (3) 434 as, (4) 444 as, (5) 454 as, (6) 464 as, (7) 474 as, (8) 482.6 as, (9) 493 as, (10) 500 as, (11) 513 as, (12) 533 as, and (13) 551.6 as; see table 2 for the magnitudes and angular locations of the maxima. 
Table 2. The location $\Delta \varphi(\tau)$ (with respect to the horizontal axis) and the magnitude, $\mathcal{W}_{-\xi}^{\xi}(\theta, \varphi=\Delta \varphi(\tau), \tau)$, of one of the two peanutshaped maxima for nine of the thirteen time delays shown in figure $6(\mathrm{f})$.

\begin{tabular}{lccccccccc}
\hline$\tau($ as $)$ & 413.6 & 424 & 434 & 444 & 464 & 482.6 & 493 & 513 & 551.6 \\
\hline$\Delta \varphi(\tau)$ & $352.8^{\circ}$ & $21.6^{\circ}$ & $43.2^{\circ}$ & $72^{\circ}$ & $122.4^{\circ}$ & $172.8^{\circ}$ & $201.6^{\circ}$ & $302.4^{\circ}$ & $360^{\circ}$ \\
$\mathcal{W}_{-\xi}^{\xi}(\theta, \varphi, \tau)$ & 1.402 & 1.391 & 1.383 & 1.377 & 1.357 & 1.336 & 1.325 & 1.312 & 1.285 \\
\hline
\end{tabular}

and the auxiliary dynamical parameters $\Upsilon_{\tau}^{\theta} \equiv \Upsilon(\theta, \tau)$ and $\Pi_{\tau}^{\theta} \equiv \Pi(\theta, \tau)$ are given by

$$
\begin{aligned}
& \mathrm{Y}_{\tau}^{\theta} \simeq \int \mathrm{d} E\left|h_{-}^{*}(\rho) A_{\Gamma_{12}}(\rho, \theta, \tau)\right| \\
& \quad \times\left\{\cos \left[\alpha_{2 s 2 p}(\rho, \theta)-\left(\epsilon_{2 s 2 p}-E_{g}\right) \tau\right]\right\}, \\
& \Pi_{\tau}^{\theta} \simeq \int \mathrm{d} E\left|h_{-}^{*}(\rho) A_{\Gamma_{12}}(\rho, \theta, \tau)\right| \\
& \quad \times\left\{\sin \left[\alpha_{2 s 2 p}(\rho, \theta)-\left(\epsilon_{2 s 2 p}-E_{g}\right) \tau\right]\right\} .
\end{aligned}
$$

Equations (28) and (29) assume that the $(2 s 2 p){ }^{1} \mathrm{P}^{o}$ doublyexcited state is the dominant intermediate state in the ionization channel $\Gamma_{12}$. Note that in the slowly-varying approximation, the interference between the $\Gamma_{12}$ and $\Gamma_{1}$ paths integrates to zero.

Plotted in figures 6(a)-(e) are the energy-integrated angular distributions in the polarization plane $(\theta=\pi / 2)$ obtained from TDSE results (i) for ${ }^{1} \mathrm{~S}^{e}$ and ${ }^{1} \mathrm{D}^{e}$ final states with $M=0, \pm 2$, and TDSE results (ii) for ${ }^{1} \mathrm{D}^{e}$ final states with $M= \pm 2$. Note that the PT formula (25) shows that the energy-integrated angular distribution, $\mathcal{W}_{\Gamma_{2}}^{\Gamma_{1}}(\theta)$, which includes only paths $\Gamma_{1}$ and $\Gamma_{2}$, does not depend on either the time delay $\tau$ or the azimuthal angle $\varphi$. Thus, for any $\tau$, plots of $\mathcal{W}_{\Gamma_{2}}^{\Gamma_{1}}(\theta)(25)$ in the polarization plane are predicted to exhibit a circular shape, as shown by our TDSE results (ii) in figures 6(a)-(e) for seven time delays. In contrast, figures 6(a)-(e) show that the energy-integrated angular distributions (i) are sensitive to both the time delay $\tau$ and the azimuthal angle $\varphi$. Indeed, as $\tau$ increases they exhibit a peanut shape that rotates with a period $T_{2 s 2 p} \simeq 69$ as. The magnitudes of the maxima of the peanut-shaped angular distributions also decreases slightly as $\tau$ increases, as shown quantitatively in table 2 and figure 6(f). All of these results are for $\phi_{12}=0$; for fixed $\tau$, varying $\phi_{12}$ would result in a global rotation of the peanut-shaped energy-integrated angular distribution (i) by an angle $\phi_{12} / 2$.

The peanut-shape of the energy-integrated angular distribution (i) shown in figures 6(a)-(e) originates from the interference between the circular angular distribution produced by the first two terms in equation (24) and the $\varphi$-dependent third term $\propto \cos \left[\chi(\theta, \tau)+\phi_{12}-2 \xi \varphi\right]$ in that equation. These interference effects are significant since each of the integrands in equations (25), (26), (28), and (29) involve dynamical parameters of comparable magnitudes, as shown in figure 5. Also, equation (24) shows that the $\phi_{12}$-dependence of the energyintegrated angular distribution (i) is controlled only by its third term, $\Lambda(\theta, \tau)\left\{2 \cos ^{2}\left[\left(\chi(\theta, \tau)+\phi_{12}\right) / 2-\xi \varphi\right]-1\right\}$. Moreover, the $\tau$-dependence of these peanut-shaped energy-integrated angular distributions (i) originates from the second and third terms of equation (24), i.e. to the pump-probe term, corresponding to path $\Gamma_{12}$, and to the term corresponding to the interference between the paths $\Gamma_{12}$ and $\Gamma_{2}$. While autoionization and QB-II are present in both the second and third terms of equation (24) (see equations (19) and (20)), the faster (attosecond) QB-I is only present in the third term of equation (24), i.e. in $\Lambda(\theta, \tau) \cos \left[\chi(\theta, \tau)+\phi_{12}-2 \xi \varphi\right]$. Specifically, the QB-I term $\left(\epsilon_{2 s 2 p}-E_{g}\right) \tau$ affects both the dynamical angle $\chi(\theta, \tau)$ (27) and the dynamical amplitude $\Lambda(\theta, \tau)$ (27) through their dependence on the auxiliary dynamical parameters $\Upsilon_{\tau}^{\pi / 2} \equiv \Upsilon(\pi / 2, \tau)(28)$ and $\Pi_{\tau}^{\pi / 2} \equiv \Pi(\pi / 2, \tau)$ (29). Note that the timescale over which the time delay $\tau$ varies in figure 6 is much shorter than the timescales for QB-II ( $\sim 1.2 \mathrm{fs})$ and for autoionization $(\sim 10 \mathrm{fs})$. Thus, on the attosecond timescale of our angular distribution results, we attribute any significant $\tau$ sensitivity of the peanut-shaped energy-integrated angular distributions (i) to attosecond QB-I dynamics. Indeed, as may be seen in figure $6(\mathrm{~d})$ and table 2 , the peanut-shaped energy-integrated angular distributions (i) for $\tau=T_{r}=413.6$ as, $\tau=T_{r}+T_{2 s 2 p} \simeq 482.6$ as, and $\tau=T_{r}+2 T_{2 s 2 p} \simeq 551.6$ as are almost the same, indicating thus a $\tau$-periodicity with a period of $\approx 69$ as. The magnitudes of the maxima of these distributions decrease slightly (about $8 \%$ ) as $\tau$ increases from 413.6 to 551.6 as (see figure 6(f) and table 2). This monotonic decrease may be due to QB-I involving other doubly-excited states besides the $(2 s 2 p){ }^{1} \mathrm{P}^{o}$ doubly-excited state, to QB-II, and/or to autoionization decay.

\subsection{Doubly-excited state effects on the energy distributions}

In the previous two sections, we demonstrated how the $2 s 2 p{ }^{1} \mathrm{P}^{o}$ doubly-excited state in path $\Gamma_{12}$ affects the fixedenergy and energy-integrated two-electron angular distributions, giving them a temporal periodicity of 69 as with the time-delay $\tau$ between the two pulses. This QB-I effect corresponds to the quantum beat period of the energy difference between the $(2 s 2 p){ }^{1} \mathrm{P}^{o}$ doubly-excited state of energy $\epsilon_{2 s 2 p}$ and the ground state of energy $E_{g}$. In this section, we demonstrate how this QB-I effect may be observed in the energy distributions of the two electrons emitted BTB in the polarization plane at a fixed azimuthal angle $\varphi=0$. We also discuss the possibility of observing the much slower QB-II beat frequencies involved between doubly-excited states.

The energy distributions for electrons detected BTB in the polarization plane at a fixed azimuthal angle $\varphi=0$ are shown in figure 7 for five time delays. (Note that these results are the same for right-left and left-right circularly polarized pulses, since according to equations (14)-(16) the dependence on the degree of circular polarization $\xi$ vanishes for $\varphi=0$.) All of these TDSE results (i) include all final state channels, ${ }^{1} \mathrm{~S}_{0}$ and ${ }^{1} \mathrm{D}$ $0, \pm 2$, in the projection (3). To illustrate changes with time delay $\tau$, all figures include the result for $\tau=T_{r}=413.6$ as. In addition, in figure 7(a) we present TDSE results (ii) that include 

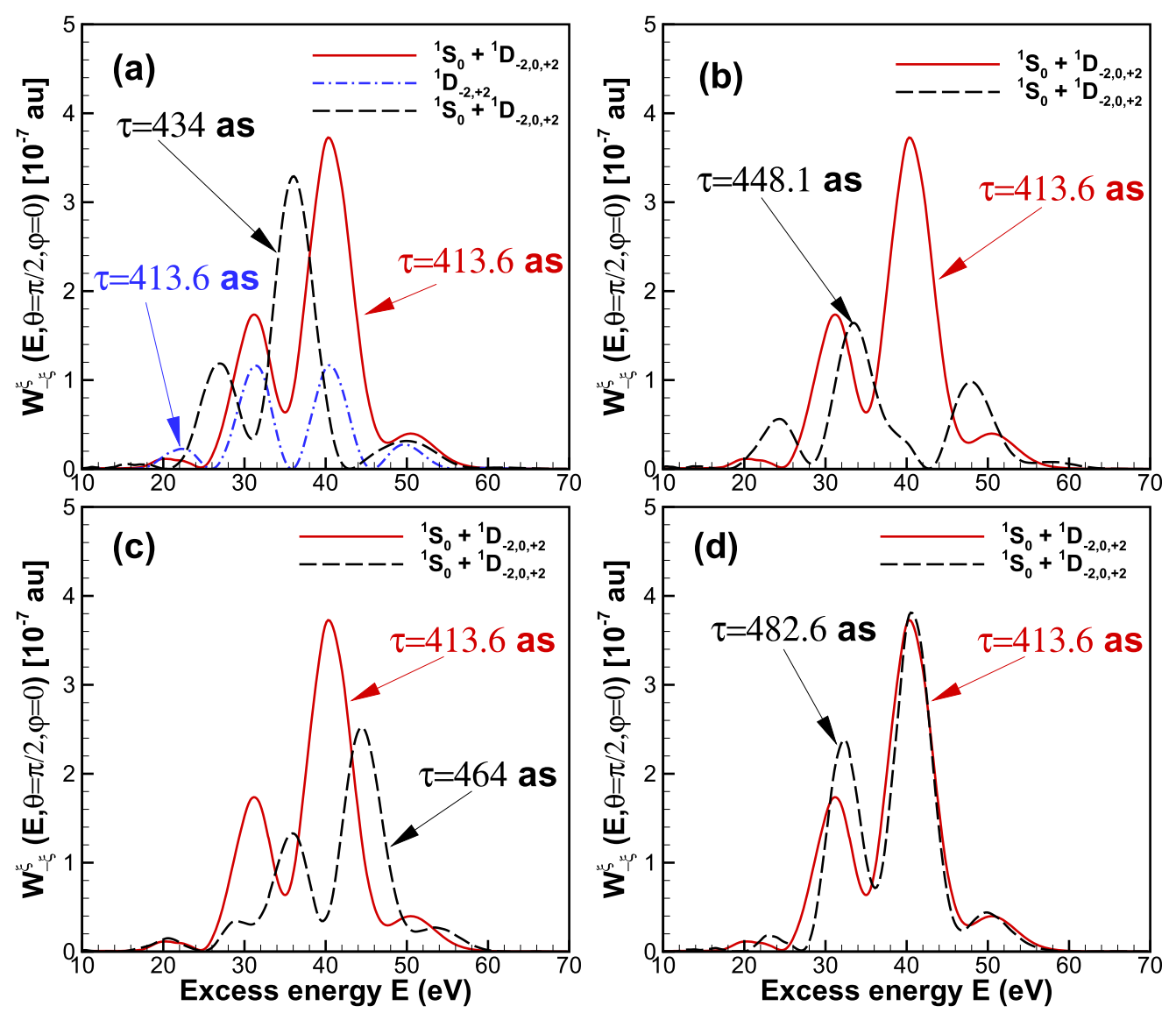

Figure 7. Energy distributions for electrons emitted BTB along the major axis of the polarization ellipse $(\theta=\pi / 2, \varphi=0)$ produced by a pair of non-overlapping right-left circularly-polarized attosecond pulses delayed in time by $\tau$, as indicated by the arrows pointing to each curve. Solid (red) curve: TDSE result (i) calculated using equation (3) for ${ }^{1} \mathrm{~S}_{0}^{e}$ and ${ }^{1} \mathrm{D}_{0, \pm 2}^{e}$ final states and analyzed in the text using equation (13) for a time delay $\tau=T_{r}=413.6$ as; this curve appears in each panel in order to serve as a reference for results for other time delays. Dashed (black) curves: TDSE results (i) for four other time delays $\tau$. Dash-dotted (blue) curve: TDSE result (ii) including only ${ }^{1} \mathrm{D}_{ \pm 2}$ final states and analyzed in the text using equation (14). In all cases, the relative energy sharing and the pulse parameters are the same as in figure 4 ; in particular, $\phi_{12}=0$.

only ${ }^{1} \mathrm{D}_{ \pm 2}$ final states for a time delay $\tau=413.6$ as. As expected from PT formula (14), this TDSE result (ii) for the energy distribution oscillates, exhibiting dark and bright Ramsey fringes, with $2 \pi / \tau$ giving the energy spacing $E_{n+1}^{\max }-E_{n}^{\max }$ between consecutive bright fringes. Indeed, the locations for maxima (bright fringes) are given by $E_{n}^{\max }=E_{g}+$ $2\left(n \pi-\phi_{12}\right) / \tau$; while those for zeros (dark fringes) are given by $E_{n}^{\text {zero }}=E_{g}+\left[(2 n+1) \pi-2 \phi_{12}\right] / \tau$, where $n$ is an integer. In figure 7(a) for $\tau=T_{r}=413.6$ as, one sees that the energy distribution (i) described by the PT formula (13) and the energy distribution (ii) described by the PT formula (14) differ significantly in both the magnitudes and the locations of maxima and minima of the energy distribution. These differences are once again the signature in the SDP (13) of both the pumpprobe signal $\Gamma_{12}$ and interference of the path $\Gamma_{12}$ with either $\Gamma_{1}$ or $\Gamma_{2}$ together with the PT term (14). With the exception of the pump-probe term in the SDP (13), all other terms (14)-(16) depend on the relative CEP $\phi_{12}$, as demonstrated in figure 8 , which shows the energy distribution (i) for three values of $\phi_{12}=0, \pi / 2$ and $\pi$.

Compared to the reference energy distribution (i) for the time delay $\tau=T_{r}=413.6$ as that appears in each panel of figure 7 , one sees that the positions of the main peak in the energy distribution (i) for other time delays $\tau$ within the first half of one QB-I period (e.g. $\tau=434$ as shown in figure 7(a) and 448.1 as shown in figure 7(b)), move to lower energies. On the other hand, for time delays $\tau$ within the second half of one QB-I period (e.g. $\tau=464$ as shown in figure 7(c)), a main peak emerges at an energy well above that of the main peak in the reference spectrum at $\tau=T_{r}=413.6$ as and moves to lower energies with increasing time delays (not shown). Then at $\tau=T_{r}+T_{2 s 2 p} \simeq 482.6$ as, figure 7(d) shows that the energy distribution (i) coincides with that at $\tau=T_{r}=413.6$ as over a broad energy range, $36 \leqslant E \leqslant 46 \mathrm{eV}$, and differs outside this range, especially for lower electron energies. (Similar results (not shown) have been obtained at $\tau=T_{r}+2 T_{2 s 2 p} \simeq 551.6$ as, with significantly larger differences found for low electron energies.) The precise periodicity of the energy distribution (i) in the vicinity of the excess energy $E=41.0 \mathrm{eV}$ of the resonant two-photon transition from the ground state to the $(2 s 2 p){ }^{1} \mathrm{P}^{o}$ doubly-excited state contrasts with the non-periodicity of the energy distributions at off-resonant excess energies. To quantify the extent of agreement in a crude way, we integrate each energy distribution in figure 7 over the energy range $10 \leqslant E \leqslant$ 
Table 3. Relative asymmetry, $\mathcal{R}(\tau)=\left[\mathcal{W}_{-\xi}^{\xi}\left(T_{r}\right)-\mathcal{W}_{-\xi}^{\xi}(\tau)\right] / \mathcal{W}_{-\xi}^{\xi}\left(T_{r}\right)$, where $T_{r}=413.6$ as, and $\mathcal{W}_{-\xi}^{\xi}(\tau)$ is the energy distribution for a time delay $\tau$ in figure 7 integrated over the energy range $10 \leqslant E \leqslant 70 \mathrm{eV}$.

\begin{tabular}{ccccccccc}
\hline$\tau$ (as) & 413.6 & 434 & 444 & 454 & 464 & 474 & 482.6 & 551.6 \\
\hline $\mathcal{R}(\tau)$ & $0 \%$ & $24.4 \%$ & $44.3 \%$ & $47.3 \%$ & $31.9 \%$ & $13.4 \%$ & $3.1 \%$ & $6.4 \%$ \\
\hline
\end{tabular}

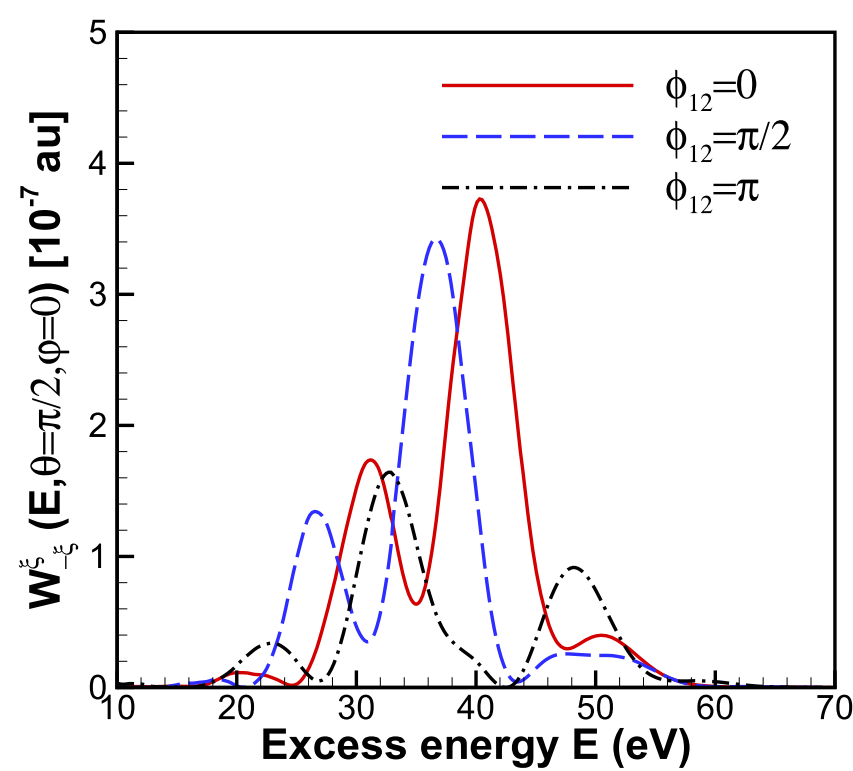

Figure 8. Sensitivity of the energy distribution, $\mathcal{W}_{-\xi}^{\xi}(E, \theta, \varphi)$, to the relative CEP $\phi_{12}$ between two counter-rotating, circularly-polarized attosecond pulses delayed by $\tau=413.6$ as, where $\theta=\pi / 2$ and $\varphi=0$. Plotted are TDSE results (i) for ${ }^{1} S_{0}$ and ${ }^{1} D_{0, \pm 2}$ final states. The detection geometry and pulse parameters are as specified in the caption of figure 4 .

$70 \mathrm{eV}$ and take the difference of the result $\mathcal{W}_{-\xi}^{\xi}(\tau)$ for a time delay $\tau$ with that for the reference time delay $\tau=$ $T_{r}=413.6$ as, $\mathcal{W}_{-\xi}^{\xi}\left(T_{r}\right)$. That is, we compute $\mathcal{R}(\tau)=$ $\left[\mathcal{W}_{-\xi}^{\xi}\left(T_{r}\right)-\mathcal{W}_{-\xi}^{\xi}(\tau)\right] / \mathcal{W}_{-\xi}^{\xi}\left(T_{r}\right)$. Table 3 shows that $\mathcal{R}(\tau)$ is smallest for time delays $\tau$ close to or equal to $n T_{2 s 2 p}, n=1,2$ (see results for $\tau=482.6$ as and $\tau=551.6$ as); while it is large for other values of $\tau$. These results again indicate a QB-I effect with periodicity of 69 as due primarily to the $(2 s 2 p){ }^{1} \mathrm{P}^{o}$ doubly-excited state in path $\Gamma_{12}$.

To eliminate the QB-I effect in the energy distributions but remain sensitive to slower kinds of doubly-excited state dynamics, we integrate the SDP (13) over the azimuthal angle $\varphi$. The result for the $\varphi$-integrated energy distribution is,

$$
\mathcal{W}_{\xi_{2}}^{\xi_{1}}(\rho, \theta, \tau)=\pi \sin ^{4} \theta\left|h_{-}(\rho)\right|^{2}+2 \pi\left|A_{\Gamma_{12}}(\rho, \theta, \tau)\right|^{2},
$$

which is independent of the relative $\operatorname{CEP} \phi_{12}$. It is comprised of only the first two terms of the SDP (13), as the interference terms (15) and (16) between the path $\Gamma_{12}$ (involving among others the faster QB-I effect) and either $\Gamma_{1}$ or $\Gamma_{2}$ vanish upon integrating over $\varphi$. As the first term in equation (30) does not depend on $\tau$, its energy distribution in the polarization plane $\left(\propto\left|h_{-}(\rho)\right|^{2}\right)$ should exhibit a Gaussian-like profile. In contrast, the second term in the PT formula (30), $\propto\left|A_{\Gamma_{12}}(\rho, \theta, \tau)\right|^{2}$, depends on the time delay $\tau$. According to equation (19), it involves autoionization decays and QB-II effects. Thus, the $\varphi$-integrated energy distribution (i) described by PT formula (30) is a unique observable for exploring only these two kinds of doubly-excited states dynamics, without the more rapid QB-I effects. Owing to the relatively long timescale $\sim 17$ fs for autoionization lifetimes and $\sim 1.2$ fs for QB-II effects, the range over which one must vary $\tau$ to map out these slow time-dependent effects is $\gtrsim 2$ fs. However, such large values of $\tau$ are beyond the capability of our current computer codes.

\section{Conclusion}

By means of both PT analyses and $a b$ initio numerical solutions of the TDSE in six spatial dimensions, we have investigated theoretically the correlated process of single-color TPDI of He by a pair of time-delayed (non-overlapping) counter-rotating, circularly-polarized attosecond pulses whose carrier frequencies are resonant with doubly excited states. This attosecond pump-probe study has focused on the detection geometry of BTB emission of electrons in the polarization plane. For nonresonant pulse carrier frequencies (such that doubly-excited states are not accessible), the momentum distributions exhibit four-arm Fermat spiral vortex patterns and the angular distributions exhibit fourfold symmetric quadrupole-like shapes. These vortices stem from an unusual kind of Ramsey interference between the two TPDI pathways, $\Gamma_{1}$ and $\Gamma_{2}$. In contrast, for resonant pulse carrier frequencies (such that ${ }^{1} \mathrm{P}^{o}$ doubly-excited states are coherently populated by one-photon transitions from the ground state induced by the first attosecond pulse), the fourfold rotational symmetry of both the vortices and the angular distribution breaks down owing to effects of the ionization pathway $\Gamma_{12}$. In fact, in the resonant case, one obtains twostart spiral vortices and twofold symmetric quadrupole-like shaped two-electron angular distributions. The change from fourfold to twofold rotational symmetry is due to interferences between the ionization pathways $\Gamma_{1}$ or $\Gamma_{2}$ with the $\tau$ sensitive ionization pathway $\Gamma_{12}$. The latter pathway involves three kinds of doubly-excited state dynamics having different timescales: QB-I ( $\sim 69$ as), QB-II ( $\sim 1.2$ fs), and autoionization decays $(\sim 17 \mathrm{fs})$.

We have predicted analytically and shown numerically that the QB-I effects due to doubly-excited states (predominantly the $(2 s 2 p){ }^{1} \mathrm{P}^{o}$ doubly-excited state) can be mapped out in various ways, including by examination of the fixed-energy two-electron angular distributions, the energyintegrated two-electron angular distributions, and the twoelectron energy distributions at a fixed emission angle. We 
have also analyzed the role played by the relative CEP $\phi_{12}$ between the two pulses for these various observables. Furthermore, we predict that the azimuthal angle-integrated energy distribution in the polarization plane, which is independent of the relative CEP, is a suitable observable to follow in real time the slower doubly-excited state dynamics, i.e. autoionization decays and QB-II effects. However, we are unable at present to provide numerical results for these slower dynamical processes.

Experimental observation of our predicted vortex patterns in the TPDI momentum distribution and the predicted doubly-excited state dynamics in the two-electron momentum, angular, and energy distributions requires the broad bandwidth of isolated, non-overlapping attosecond pulses. Control of the relative CEPs and the polarizations of the attosecond pulse pairs is also essential. Reaction microscope techniques [39] already exist for measuring TPDI momentum distributions.

\section{Acknowledgments}

This work is supported in part by the US Department of Energy (DOE), Office of Science, Basic Energy Sciences (BES), Grant No. DE-FG02-96ER14646. Our computations were conducted using the Sandhills and Crane computing facilities of the Holland Computing Center at the University of Nebraska, and as well as the Stampede supercomputer (TACC) under US National Science Foundation Grant No. PHY-120003.

\section{ORCID iDs}

J M Ngoko Djiokap (ib https://orcid.org/0000-00034702-3544

Anthony F Starace (iD https://orcid.org/0000-00017261-9329

\section{References}

[1] Paul P M, Toma E S, Breger P, Mullot G, Augé F, Balcou P, Muller H G and Agostini P 2001 Observation of a train of attosecond pulses from high harmonic generation Science 2921689

[2] Hentschel M, Kienberger R, Spielmann C, Reider G A, Milosevic N, Brabec T, Corkum P, Heinzmann U, Drescher M and Krausz F 2001 Attosecond metrology Nature 414509

[3] Sansone G et al 2006 Isolated single-cycle attosecond pulses Science 314443

[4] Goulielmakis E et al 2008 Single-cycle nonlinear optics Science 3201614

[5] Zhao K, Zhang Q, Chini M, Wu Y, Wang X and Chang Z 2012 Tailoring a 67 attosecond pulse through advantageous phase-mismatch Opt. Lett. 373891

[6] Krausz F and Ivanov M 2009 Attosecond physics Rev. Mod. Phys. 81163
[7] Kling M F and Vrakking M J 2008 Attosecond electron dynamics Annu. Rev. Phys. Chem. 59463

[8] Tzallas P, Skantzakis E, Nikolopoulos L A A, Tsakiris G D and D Charalambidis D 2011 Extreme-ultraviolet pumpprobe studies of one-femtosecond-scale electron dynamics Nat. Phys. 7781

[9] Sansone G, Poletto L and Nisoli M 2011 High-energy attosecond light sources Nat. Photon. 5655

[10] Hu S X and Collins L A 2006 Attosecond pump probe: exploring ultrafast electron motion inside an atom Phys. Rev. Lett. 96073004

[11] Morishita T, Watanabe S and Lin C D 2007 Attosecond light pulses for probing two-electron dynamics of helium in the time domain Phys. Rev. Lett. 98083003

[12] Feist J, Nagele S, Ticknor C, Schneider B I, Collins L A and Burgdörfer J 2011 Attosecond two-photon interferometry for doubly excited states of helium Phys. Rev. Lett. 107 093005

[13] Jiang W-C, Xiong W-H, Zhu T-S, Peng L-Y and Gong Q 2014 Double ionization of He by time-delayed attosecond pulses J. Phys. B 47091001

[14] Medišauskas L, Wragg J, van der Hart H and Ivanov M Y 2015 Generating isolated elliptically polarized attosecond pulses using bichromatic counterrotating circularly polarized laser fields Phys. Rev. Lett. 115153001

[15] Hernández-García C, Durfee C G, Hickstein D D, Popmintchev T, Meier A, Murnane M M, Kapteyn H C, Sola I J, Jaron-Becker A and Becker A 2016 Schemes for generation of isolated attosecond pulses of pure circular polarization Phys. Rev. A 93043855

[16] Ngoko Djiokap J M, Manakov N L, Meremianin A V, Hu S X, Madsen L B and Starace A F 2014 Nonlinear dichroism in back-to-back double ionization of $\mathrm{He}$ by an intense elliptically polarized few-cycle extreme ultraviolet pulse Phys. Rev. Lett. 113223002

[17] Ngoko Djiokap J M, Hu S X, Madsen L B, Manakov N L, Meremianin A V and Starace A F 2015 Electron vortices in photoionization by circularly polarized attosecond pulses Phys. Rev. Lett. 115113004

[18] Yuan K-J, Chelkowski S and Bandrauk A D 2016 Photoelectron momentum distributions of molecules in bichromatic circularly polarized attosecond UV laser fields Phys. Rev. A 93053425

[19] Ngoko Djiokap J M, Meremianin A V, Manakov N L, Hu S X, Madsen L B and Starace A F 2016 Multistart spiral electron vortices in ionization by circularly polarized pulses Phys. Rev. A 94013408

[20] Yuan K-J, Lu H and Bandrauk A D 2017 Photoionization of triatomic molecular ions $\mathrm{H}_{3}^{2+}$ by intense bichromatic circularly polarized attosecond UV laser pulses J. Phys. B 50 124004

[21] Ngoko Djiokap J M, Hu S X, Meremianin A V, Manakov N L, Madsen L B and Starace A F 2017 Kinematical vortices in double photoionization of helium by attosecond pulses Phys. Rev. A 96013405

[22] Pengel D, Kerbstadt S, Johannmeyer D, Englert L, Bayer T and Wollenhaupt M 2017 Electron vortices in photoionization by circularly polarized attosecond pulses Phys. Rev. Lett. 118053003

[23] Pindzola M S and Robicheaux F 1996 Total ionization cross section for electron-hydrogen scattering using a timedependent close-coupling method Phys. Rev. A 542142

[24] Pindzola M S and Robicheaux F 1998 Time-dependent closecoupling calculations of correlated photoionization processes in helium Phys. Rev. A 57318

[25] McCurdy C W, Baertschy M and Rescigno T N 2004 Solving the three-body Coulomb breakup problem using exterior complex scaling J. Phys. B 37 R137 
[26] Hu S X 2010 Optimizing the FEDVR-TDCC code for exploring the quantum dynamics of two-electron systems in intense laser pulses Phys. Rev. E 81056705

[27] Ngoko Djiokap J M, Hu S X, Jiang W-C, Peng L-Y and Starace A F 2012 Enhanced asymmetry in few-cycle attosecond pulse ionization of $\mathrm{He}$ in the vicinity of autoionizing resonances New J. Phys. 14095010

[28] Ngoko Djiokap J M, Hu S X, Jiang W-C, Peng L-Y and Starace A F 2013 Asymmetries in production of $\mathrm{He}^{+}(n=2)$ with an intense few-cycle attosecond pulse Phys. Rev. A 88 011401

[29] Ngoko Djiokap J M, Manakov N L, Meremianin A V and Starace A F 2013 Carrier-envelope-phase-induced asymmetries in double ionization of helium by an intense few-cycle XUV pulse Phys. Rev. A 88053411

[30] Muller H G 1999 An efficient propagation scheme for the timedependent Schrödinger equation in the velocity gauge Laser Phys. 9138

[31] Kjeldsen T K, Nikolopoulos L A A and Madsen L B 2007 Solving the $\mathrm{m}$-mixing problem for the three-dimensional time-dependent Schrödinger equation by rotations: application to strong-field ionization of $\mathrm{H}_{2}^{+}$Phys. Rev. A 75 063427
[32] Madsen L B, Nikolopoulos L A A, Kjeldsen T K and Fernández J 2007 Extracting continuum information from $\Psi(t)$ in time-dependent wave-packet calculations Phys. Rev. A 76063407

[33] Foumouo E, Antoine P, Bachau H and Piraux B 2008 Attosecond timescale analysis of the dynamics of twophoton double ionization of helium New J. Phys. 10025017

[34] Dörner R et al 1996 Fully differential cross sections for double photoionization of He measured by recoil ion momentum spectroscopy Phys. Rev. Lett. 771024

[35] Feagin J M 1996 Recoil-ion angular distributions following double photoionization J. Phys. B 29 L551

[36] Walter M, Meremianin A V and Briggs J S 2003 Multi-particle photoionization by a single photon $J$. Phys. B 364561

[37] Liu C-N, Chen M-K and Lin C D 2001 Radiative decay of helium doubly excited states Phys. Rev. A 64010501

[38] Iemura $\mathrm{K}$ et al 2001 Electron spectroscopy of doubly excited states in He produced by slow collisions of $\mathrm{He}^{2+}$ ions with Ba atoms Phys. Rev. A 64062709

[39] Ullrich J, Moshammer R, Dorn A, Dörner R, Schmidt L P H and Schmidt-Böcking H 2003 Recoil-ion and electron momentum spectroscopy: reaction-microscopes Rep. Prog. Phys. 661463 